\title{
Monitoring Groundwater and River Interaction Along the Hanford Reach of the Columbia River
}

M. D. Campbell

April 1994

Prepared for the U.S. Department of Energy under Contract DE-AC06-76RLO 1830

Pacific Northwest Laboratory

Richland, Washington 99352 


\section{Executive Summary}

As an adjunct to efficient Hanford Site characterization and remediation of groundwater contamination, an automatic monitor network has been used to measure Columbia River and adjacent groundwater leveis in several areas of the Hanford Site sizce 1991. Water levels, temperatures, and electrical conductivity measured by the automatic nonitor network provided an initial database with which to calibrate models and from which to infer ground and river water interactions for site characterization and remediation activities. Measurements of the dynamic river/aquifer system have been simultaneous at 1-hr intervals, with a quality suitable for hydrologic modeling and for computer model calibration and testing. This report describes the equipment, procedures, and results from measurements done in 1993.

During 1993, Columbia River and groundwater elevations were measured hourly at 50 locations in seven areas of the Hanford Site in southcentral Washington State. Water temperature was measured at 10 of these locations; electrical conductivity was measured at five.

Water level accuracy is unknown, but is believed to be within $\pm 0.1 \mathrm{ft}$. Factors affecting accuracy are topographic surveys, instrument calibrations, and steel tape measurements. Measurement precision appears to be within $\pm 0.02 \mathrm{ft}$ for wells and $\pm 0.2 \mathrm{ft}$ for river stage. Repeated steel tape measurements vary within $\pm 0.01 \mathrm{ft}$. Survey errors vary with distance between sites, but most appear to be less than $\pm 0.07 \mathrm{ft}$.

Visual inspection and graphic review of data confirmed its continuity. Comparisons between simultaneous steel tape and datalogger readings were used as periodic data quality checks. River elevations varied up to $15 \mathrm{ft}$ during the year and up to $9 \mathrm{ft}$ during a single day. The 300 Area river elevation varied about $60 \%$ as much as the other stations because of the influence of the McNary Dam forebay. Ice Harbor Dam tailwater also influenced the fluctuation of the river in the 300 Area. Groundwater elevations varied up to about $3 \mathrm{ft}$ per day in a few wells nearest the river and up to about $6 \mathrm{ft}$ over the season in some wells.

Electrical conductivity of water in wells was influenced by river intrusion to a small degree. Riverbank seep conductivity varied between river and well values. Each of these factors is important in more efficient site characterization and remediation. 


\section{Contents}

Executive Summary $\ldots \ldots \ldots \ldots \ldots \ldots \ldots \ldots \ldots \ldots \ldots \ldots \ldots \ldots \ldots$ iii

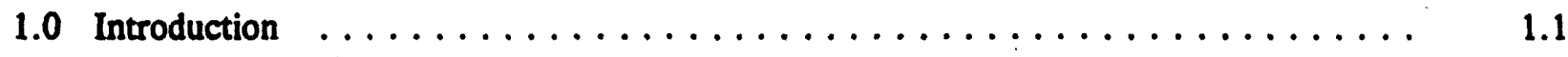

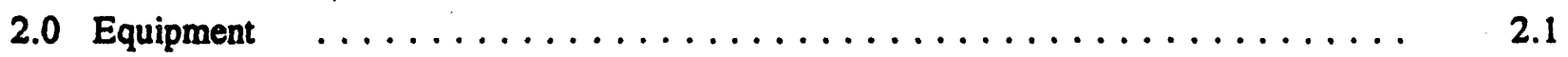

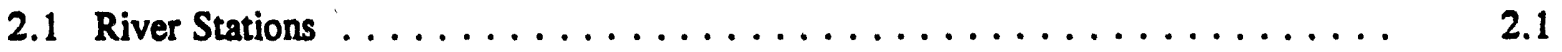

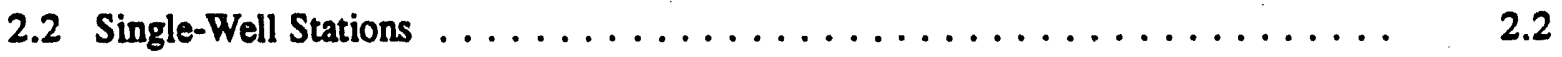

2.3 Multiple-Well Stations . . . . . . . . . . . . . . . . . 2.2

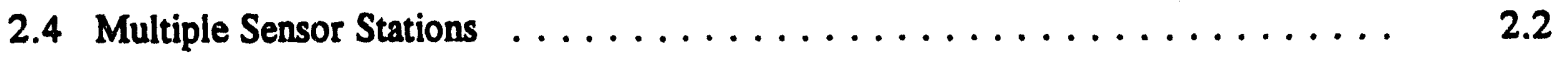

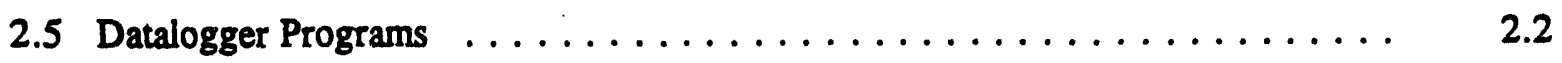

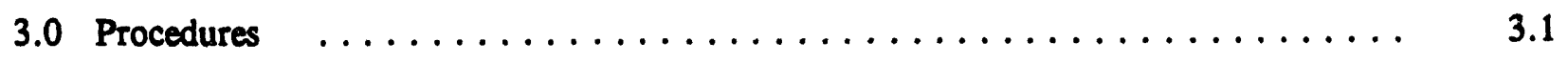

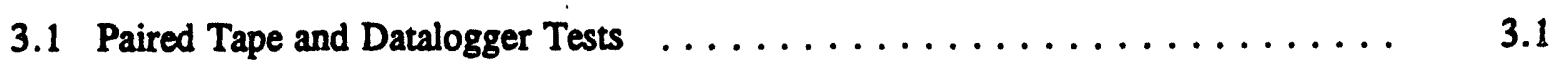

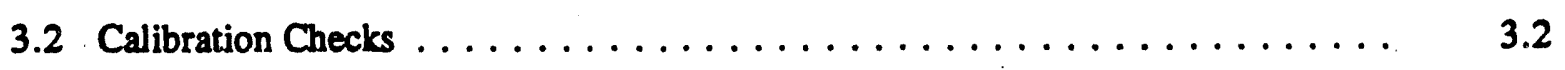

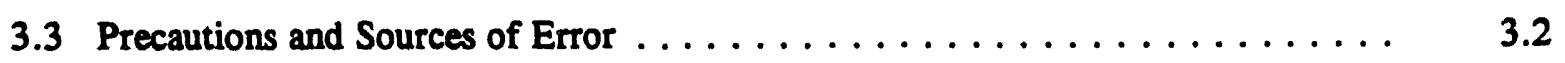

3.4 Desiccant and Battery Service . . . . . . . . . . . . . . . 3.4

4.0 Results and Conclusions $\ldots \ldots \ldots \ldots \ldots \ldots \ldots \ldots \ldots \ldots \ldots \ldots \ldots \ldots$

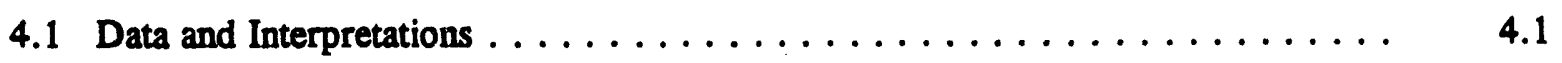

4.2 Conclusions $\ldots \ldots \ldots \ldots \ldots \ldots \ldots \ldots \ldots \ldots \ldots \ldots \ldots \ldots \ldots \ldots$

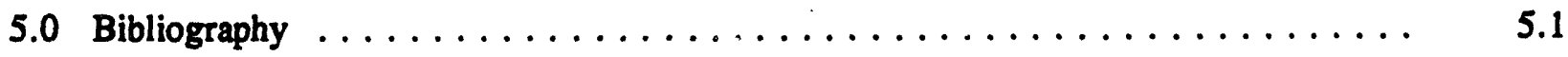

Appendix A - Datalogger Programs $\ldots \ldots \ldots \ldots \ldots \ldots \ldots \ldots \ldots \ldots \ldots \ldots \ldots$

Appendix B - Steel Tape and Dataloggers Paired Sets $\ldots \ldots \ldots \ldots \ldots \ldots \ldots \ldots \ldots$. . . . . .

Appendix C - Location and Calibration Data $\ldots \ldots \ldots \ldots \ldots \ldots \ldots \ldots \ldots \ldots \ldots$ 


\section{Figures}

1. Network Monitor Station Locations During $1993 \ldots \ldots \ldots \ldots \ldots \ldots$

2. Columbia River Stage at Five Stations $\ldots \ldots \ldots \ldots \ldots \ldots \ldots \ldots \ldots \ldots \ldots \ldots$

3. Columbia River Stage at Four Stations for 25 Days . . . . . . . . . . . . . 4.3

4. Columbia River Stage at Five Stations for Five Days, Shoring Time Lag and Attenuation $\ldots \ldots \ldots \ldots \ldots \ldots \ldots \ldots \ldots \ldots \ldots \ldots \ldots \ldots$

5. Columbia River Stage at Four Stations During 10 Days of Low Flow . . . . . . . . 4.5

6. Water Elevations in Three Wells and The River in The 300 Area . . . . . . . . . 4.6

7. Detailed Variations in Water Elevations in The 300 Area $\ldots \ldots \ldots \ldots \ldots . \ldots$

8. Water Elevations in Three Wells and The River at $100-\mathrm{F}$ Area . . . . . . . . . 4.8

9. Detailed Variations in Water Elevations in 100-F Area . . . . . . . . . . . 4.9

10. Water Elevations in Three Wells and The River in $100-\mathrm{H}$ Area $\ldots \ldots \ldots \ldots \ldots . \ldots .11$

11. Detailed Variations in Water Elevations in $100-\mathrm{H}$ Area $\ldots \ldots \ldots \ldots \ldots \ldots . \ldots . . \ldots$

12. Water Elevations in 100-D and 100-K Wells and The 100-B River . . . . . . 4.13

13. Detailed Variations of 100-D and 100-K Wells and The 100-B River . . . . . . . . 4.14

14. Water Elevations at $100-B$ Area $\ldots \ldots \ldots \ldots \ldots \ldots \ldots \ldots \ldots \ldots \ldots$

15. Detailed Variations in Water Elevations in 100-B Area . . . . . . . . . . . 4.16

16. Electrical Conductivity of $100-\mathrm{H}$ Seeps, Well, and River $\ldots \ldots \ldots \ldots \ldots \ldots \ldots$

17. Detailed Variation of Electrical Conductivity at $100-H$ Area $\ldots \ldots \ldots \ldots \ldots \ldots$

18. Thermal Variations of River and Groundwater at $100-\mathrm{H}$ Area . . . . . . . . . 4.20

\section{Tables}

1. Datalogger Range and System Resolution, Assuming Calibration Factor of $0.93 \mathrm{ft}$ volt-ratio $\ldots \ldots \ldots \ldots \ldots \ldots . \ldots . \ldots . . \ldots$

2. Paired Sets of Tape and Datalogger Readings . . . . . . . . . . . . 3.3 


\subsection{Introduction}

Aquifer hydraulic properties and head gradients control contaminant migration to the Columbia River. Contaminant migration also depends on concentration and thermal gradients operating in the aquifer/river system. Properly calibrated computer models can process such data to simulate interactions between the unconfined aquifer and the Columbia River to show likely consequences of remediation. Measurement variety, frequency, and accuracy must satisfy model requirements for acceptable predictive reliability.

The monitor network is a feasible and economical system of collecting the simultaneous and frequent data required for remedial investigation of river and groundwater interaction. Monitors collect the data automatically and transmit it by radio telemetry to a computer for storage and processing.

During 1993, river and groundwater levels were measured by an automatic monitor network at 50 locations in seven areas along the Columbia River on the Hanford Site. Water temperatures at 10 locations and electrical conductivity at five locations were also measured. Pacific Northwest Laboratory (PNL) $)^{(a)}$ personnel, under contract to Westinghouse Hanford Company (WHC), initiated network installation early in FY 1991 at 35 sites in the 300 Area, with nine sites added later that year in 100-F, $-\mathrm{H}$, and $-\mathrm{B}$ Areas.

Emphasis and equipment were shifted from the 300-FF-5 Operable Unit to the 100 Aggregate Area Operable Unit during the spring of 1993. Monitors removed from the 300 Area were installed in $100-D,-N$, and $-\mathrm{K}$ Areas and added to the number in the $-\mathrm{F},-\mathrm{H}$, and $-\mathrm{B}$ Areas. Eleven wells and one river station are still monitored in the 300-FF-5 boundary while the others are spread about evenly among the 100 Areas. The monitor network currently consists of 44 radio transceivers and 42 automatic dataloggers, with one station in each area, except $100-\mathrm{N}$, serving two wells. The seven areas currently monitored are shown in Figure 1.

This report summarizes conductivity, temperature, and water level data obtained by the network. Equipment and procedures are detailed. The monitor stations are discussed. Quality and calibration are discussed, followed by measurements and the programs used to make them. Electrical conductivity, temperatures, and water levels are presented and discussed. Finally, measurements and their frequency and precision are presented and discussed, and conclusions are presented. Network programming and other information are presented in the appendixes.

(a) Operated by Battelle Memorial Institute for the U.S. Department of Energy under Contract DE-ACO6-76RLO 1830. 


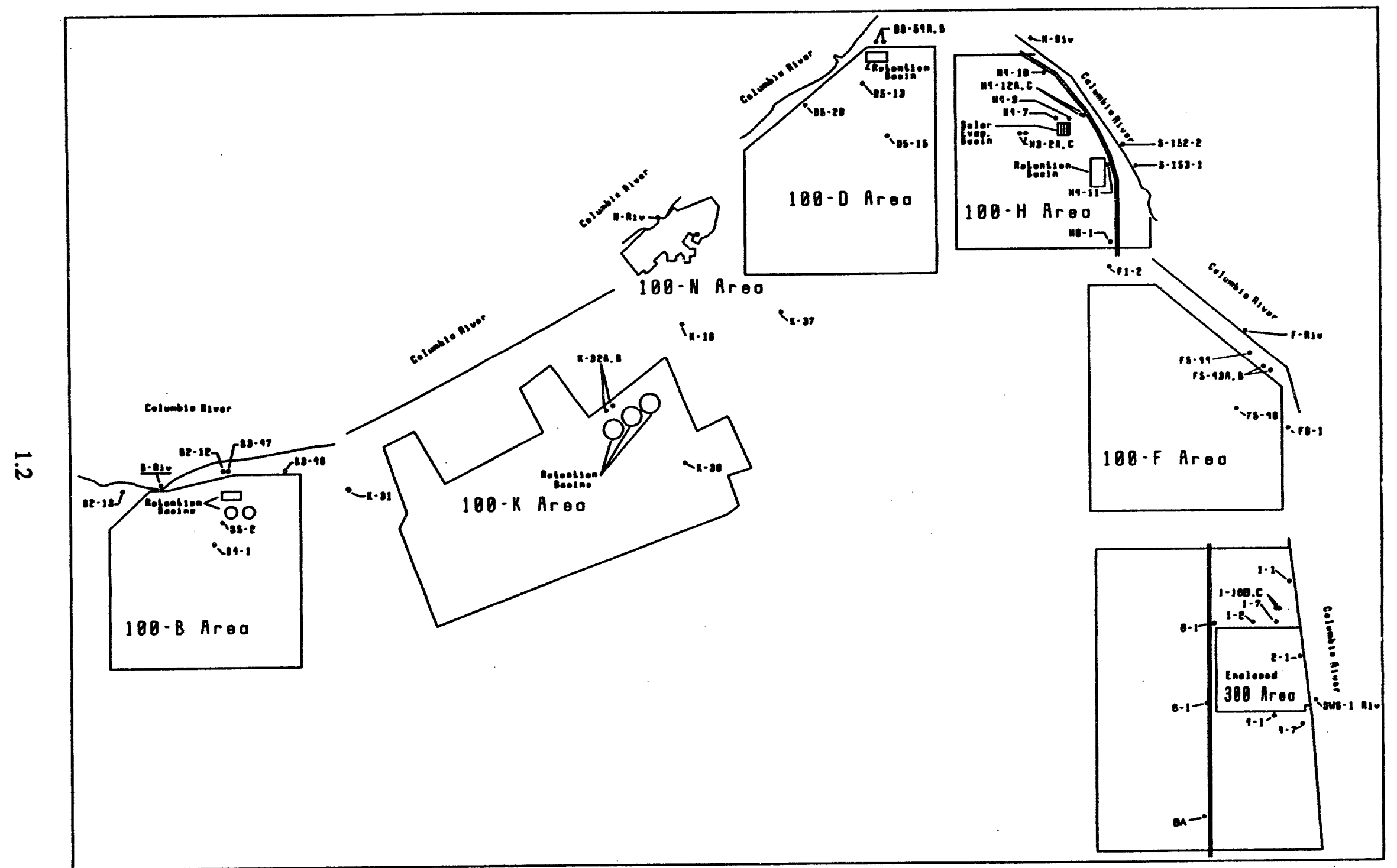

Figure 1. Network Monitor Station Locations During 1993 


\subsection{Equipment}

Monitor network stations have a datalogger, a frequency modulated (FM) radio transceiver with a radio frequency (RF) modem connected to the datalogger, an FM antenna, and a 12-volt battery with a solar recharging panel. The datalogger, modem, and transceiver are installed in a weather enclosure. The entire assembly is mounted on a tripod or a well post pipe. Forty-eight of the $\mathbf{5 0}$ sites have pressure transducers to measure water depth. Ten sites have thermocouples to measure water temperatures. Five sites have probes to sense electrical conductivity and water temperature. The electrical conductivity measurements, corrected for temperature, reflect the concentration of dissolved ions.

\subsection{River Stations}

Two of the five river stations have the transducer retainer pipe extending down the river bank slope into the water at an angle. One station is staked to the bank and is likely to move with time. These two stations, located at 100-F and 100-H, require adjustments to the steel tape measurements taken along the pipe to compensate for their non-vertical angle. The 100-F correction is $0.2425 \times$ tape reading. The $100-\mathrm{H}$ correction was $0.4395 \times$ tape reading up to January 25,1994 and $0.4417 \times$ tape reading thereafter. The other three stations, 100-B, 100-N, and SWS-1 in the 300 Area, have vertically mounted transducer retainer pipes. SWS-1 in the 300 Area has a river stage scale mounted on the concrete wall next to the retainer pipe. Although river stage can be read directly from the scale, survey indicated the need for a $0.4 \mathrm{ft}$ adjustment. The $100-\mathrm{N}$ and 100-B stations require steel tape measurements from the top of the top pipe coupling to the water surface.

All river station elevations were surveyed. The SWS-1 scale was reported to be $0.4 \mathrm{ft}$ lower than its indicated value. Thus, the 345-ft stage scale mark is actually $344.6 \mathrm{ft}$ above mean sea level (MSL). The 100-F river station surveyed as $373.33 \mathrm{ft}$ above MSL at the top, south corner of the channel iron. The bottom south corner of the channel was $368.48 \mathrm{ft}$ above MSL. The 100-H river station was originally surveyed as $386.47 \mathrm{ft}$ above MSL at the top of the 2-in. pipe coupling; resurvey on January 25, 1994 set the elevation at $386.83 \mathrm{ft}$ atove MSL. The $100-\mathrm{N}$ river station surveyed as $426.09 \mathrm{ft}$ above MSL at the top of the pipe coupling. The 100-B river station surveyed as $448.32 \mathrm{ft}$ above MSL at the top of the pipe coupling.

The 100-H river station has an electrical conductivity cell as well as a pressure transducer attached. This station measured depth, electrical conductivity, and temperature of the water. Water depth was recorded as a feet-per-volt ratio, ranging between 0 . and 15.xxxx. Electrical conductivity was recorded as milliSiemens, ranging from 0.0000 to $0.5 \times x x$. Electrical conductivity before October 5 , 1993 required temperature compensation during data processing, but it was compensated in the datalogger after that date. Temperature ranged from 0 to $30^{\circ} \mathrm{C}$. 


\subsection{Single-Well Stations}

Single-well stations have transducers connected to channels 1 and 2 of the current dataloggers, with orange, black, yellow, and blue wires connected to $1 \mathrm{H}, 1 \mathrm{~L}, 2 \mathrm{H}$, and $2 \mathrm{~L}$, respectively. The red wire is connected to the El excitation channel and the white wire connected to any AG. The silver wire is not connected because of its tendency to create a ground loop.

\subsection{Multiple-Well Stations}

Multiple-well stations have transducers connected sequentially. The transducer from the first well, numerically or alphabetically, is connected as described for the single well station. The second transducer is connected with orange, black, yellow, and blue going to $3 \mathrm{H}, 3 \mathrm{~L}, 4 \mathrm{H}$, and $4 \mathrm{~L}$, respectively. The red wire is connected to E1 with the first transducer, and the white wire is connected to any AG terminal.

The readout from multiple-well stations is in the sequence wired. Early in the monitor project, some well numbers were unknown when we began monitoring them. In some cases, the sequence was reversed, causing difficulty in quality control. This problem required help from the Database Manager in the Geosciences Department of WHC or reference to the Laboratory Record Book to avoid errors in data interpretation.

\subsection{Multiple Sensor Stations}

Pressure, temperature, and electrical conductivity of water were measured in some locations. Stations that measured all three were wired to measure pressure, electrical conductivity, and temperature, in that order. The electrical conductivity cell has six wires. Either the blue or the black wire can be used, but not both. The black wire is used when high resolution is required. The connections used were red-6L, white-6H, green-E3, black-E2, purple-AG, and clear-G. This configuration avoids interference and allows for use of two pressure transducers on a single datalogger.

Where electrical conductivity is measured, temperature is also measured by the same transducer and used to compensate for thermal changes in the river or groundwater solution.

\subsection{Datalogger Programs}

The transducers used in our monitor systems require precise, selectable excitation, and simultaneous measurements of transducer excitation and output. The datalogger programs to accomplish this task are shown in Appendix A. 


\subsection{Procedures}

Periodic maintenance and tests were required to assure quality data through proper equipment operation. Tape and datalogger readings recorded simultaneously were compared with previously recorded sets to detect changes. Errors and problems were addressed. Initial calibration and recalibration determined the factors for data reduction. Battery voltage was measured periodically, and desiccant packages were replaced to help ensure proper equipment operation.

\subsection{Paired Tape and Datalogger Tests}

Water levels at a site were simultaneously measured by steel tape and by a datalogger. Readings were repeated when paired reading sets disagreed with previously paired reading sets.

An equivalent change in water level is required by the tape and the datalogger. The following equation was used to deal with errors:

$$
T_{1}-T_{2}=f\left(D L_{1}-D L_{2}\right)
$$

where $T_{1}$ and $T_{2}$ are steel tape readings 1 and $2, " f "$ is the calibration factor for the transducer, and $\mathrm{DL}_{1}$ and $\mathrm{DL}$ are the paired datalogger readings 1 and 2.

Dataloggers measured volt ratios as transducer voltage output/input. The following equation converts the datalogger reading into elevation relative to MSL elevation:

$$
E_{\text {MSL }}=E_{T O C}-T+\left(D L-D L_{T}\right) \times F
$$

$$
\text { where } \begin{aligned}
& \mathrm{E}_{\mathrm{MSL}}=\text { elevation above Mean Sea Level } \\
& \mathrm{E}_{\mathrm{TOC}}=\text { surveyed elevation at top of the well casing } \\
& \mathrm{T}=\text { steel tape measurement } \\
& \mathrm{DL}=\text { ambient datalogger reading } \\
& \mathrm{DL} / \mathrm{datalogger} \text { reading taken with the tape reading } \\
& \mathrm{F}=\text { the calibration factor for the transducer. }
\end{aligned}
$$

All pressure transducer readings were preserved as volt ratios. Calibration factors were not stored with raw data. Raw data were preserved in computers in two separate locations, readily accessible, for processing.

Datalogger resolution is $0.33 \mu \mathrm{V}$ on its most sensitive range, which corresponds with $0.000123 \mathrm{ft}$ of water elevation change. Both voltage and depth resolution are displayed in Table 1. 
Table 1. Datalogger Range and System Resolution, Assuming Calibration Factor of $0.93 \mathrm{ft} /$ volt-ratio

\begin{tabular}{|c|c|c|c|}
\hline \multicolumn{3}{|c|}{ Datalogger } \\
\cline { 1 - 2 } Conse & $\begin{array}{c}\text { Sensitivity } \\
(\mathrm{mv})\end{array}$ & $\begin{array}{c}\text { System Resolution } \\
(\mathrm{ft})\end{array}$ \\
\hline 21 & 2.5 & 0.00033 & 0.0001 \\
\hline 22 & 7.5 & 0.00100 & 0.0003 \\
\hline 23 & 25. & 0.00333 & 0.0012 \\
\hline 24 & 250. & 0.0333 & 0.012 \\
\hline 25 & 2500. & 0.333 & 0.12 \\
\hline
\end{tabular}

\subsection{Calibration Checks}

Cross-checking the datalogger readings with the steel tape measurements helped identify errors. Errors have been traced to well casing extension, misread tape, transducer movement, transducer failure, and datalogger wiring panel failure. Table 2 shows examples of correct and erroneous tape and datalogger reading sets. (See Appendix B for all reading sets.) Some well casings were extended upward approximately $1 \mathrm{ft}$ when some of the older wells were renovated. When the work was done, a time lag occurred before resurvey records were available, so the water elevations appeared incorrect until resurvey.

Steel tape measurements are commonly accepted as the standard, but tape reading accuracy depends on technique, individual observation, and weather conditions. For example, tape may be lodged on an obstacle rather than suspended straight into the water. Detection of this problem depends on the observer's skill and judgment. Also, the wet line across the tape scale may result from either normal water submergence or from contact with a condensing surface and may also require the observer's judgment. Repeated tape measurements revealed observer errors ranging up to $10 \mathrm{ft}$. Errors were more difficult to detect and resolve in wells with other equipment. Large amounts of condensation near the top of the well casing made accurate measurement more difficult. However, we repeated measurements to verify the steel tape readings within $0.01 \mathrm{ft}$. Disagreement between steel tape and transducer readings required repeat readings to achieve consistency. Steel tape readings should be questioned and proven by replicated remeasurement.

\subsection{Precautions and Sources of Error}

Errors in Table 2 and Appendix B include those that are positive because a steel tape or datalogger reading was too small. Errors that are negative result from a steel tape or datalogger reading that is too large. Condensate wetting of a steel tape causes a positive error. Transducer cable slippage and steel tape hangup cause a negative error. Aquifer changes from wind cause both positive and negative 
Table 2. Paired Sets of Tape and Datalogger Readings (see Appendix B)

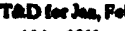

cot Mer $1 \mathrm{me}$

\begin{tabular}{|c|c|c|c|c|c|c|c|c|c|c|c|c|c|c|c|c|c|}
\hline Tap in & & $T$ & & & & & & & & & & & & & & & \\
\hline Dew & & Tan & ocenese & $\begin{array}{l}\text { Cones } \\
\text { (il) }\end{array}$ & $\begin{array}{l}\text { Wall } \\
\text { Dave }\end{array}$ & $n=0$ & rep & netace & (i) & $\begin{array}{l}\text { Wall } \\
\text { Daw }\end{array}$ & 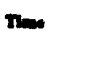 & Top & aces & $\begin{array}{l}\text { Claces } \\
\text { (in) }\end{array}$ & Wall & $\operatorname{nim}$ & Taw \\
\hline $1-1$ & & & & & 1.7 & & & & & 29 & & & & & 49 & & \\
\hline $1-20 x$ & 97 & Mes & casso & .00 & 1.99 & 1450 & 12.41 & 3,1228 & acs & eis-9 & 1000 & $\Delta n$ & 02000 & 000 & 2isen & 104 & 37.25 \\
\hline $25 x$ & 1137 & uns & 20525 & .0 .12 & 2.s.se & 1030 & ass & 1.900 & $a$ & 429 & 1217 & 40 & Land & 000 & 220 & 1211 & 320 \\
\hline 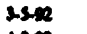 & 100 & 340 & Lssio & a.13 & 25.92 & 1013 & 46 & 2.191 & 000 & $\sin x$ & 736 & 150 & 3002 & an & $\operatorname{sen}$ & 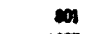 & $\operatorname{san}$ \\
\hline $46 x$ & $\infty$ & 3420 & WrDO & a.13 & $42^{4} 6$ & sor & ass & $2 \times 0 \times$ & 46 & 1299 & 2011 & 4031 & 3.1100 & ast & 12.99 & 1000 & $\omega_{12}$ \\
\hline$s \in x$ & 736 & 330 & Sum & 000 & 421.92 & I335 & Ma.ss & $4 \pi 2$ & & $1.9 x$ & 1021 & Mus & 10742 & and & $1.9 n$ & 140 & 3250 \\
\hline $6-x$ & 72 & 1200 & $\operatorname{sen} 6$ & 200 & 0.3 .92 & 712 & 0223 & 72360 & act & 22.92 & 1010 & $\omega n$ & 21700 & Ad & 25.20 & 1066 & 100 \\
\hline $7.28 \Omega$ & $n i$ & $\operatorname{sen}$ & 2ssen & arr & 7.2 .92 & 740 & 12.37 & 7.1209 & 000 & 369 & 1125 & 466 & $\mathbf{2 4 6 7 2}$ & ane & 1628 & IIIs & acs \\
\hline $2 .-92$ & $m s$ & $\mathbf{M n g}$ & 36517 & aos & 27.92 & $n 3$ & 421 & s.ten & an & 492 & 260 & 1625 & 2710 & 000 & 409 & 957 & 4034 \\
\hline $\operatorname{sen} 2$ & 910 & 3SAS & 30012 & a19 & 29.92 & $7 \mathbf{4 0}$ & 467 & cons & aOR & $\sin x$ & 1234 & 4500 & $\cos 2$ & ov & $\operatorname{sis} 20$ & 12212 & 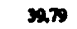 \\
\hline 1ates & $\infty$ & 3520 & 36006 & an & 10.en & DS & 44.47 & SeA6 & and & 6.2 .92 & 1219 & 1500 & 50000 & 0000 & 6.2 .92 & 1223 & $\operatorname{sen}$ \\
\hline $11-10.92$ & 20 & 33.14 & 32406 & 0.32 & $11-10-92$ & $\$ 27$ & $\mathbf{w}$ & 50819 & aOr & $\cos 2 n$ & 11ss & Mr & $5 \mathrm{sen}$ & 030 & $\cos 0 x$ & $11 n$ & 32.75 \\
\hline $12+\infty 2$ & 95 & 33.12 & coeses & .032 & $12+92$ & 911 & 124 & 70000 & +000 & $7+92$ & 200 & 17.36 & 24510 & aor & $\cos 2$ & $m$ & 41. $n$ \\
\hline 1.7 .83 & $m$ & 32.32 & CQDO1 & $a z$ & 1.7 .90 & (1) & 1100 & 7.9452 & $a$ & $26 \cdot 2$ & 736 & 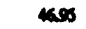 & 20004 & and & 2en & 12000 & 41,47 \\
\hline 2920 & 200 & 35.19 & 3nn & ars & 200 & 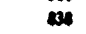 & $\mathbf{m u}$ & $\omega n$ & 000 & 2092 & 1200 & 8.37 & 20057 & wer & $100 x$ & 1316 & MLAS \\
\hline 290 & 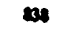 & 3502 & 2012 & $a 23$ & $3-90$ & ns & $\mathrm{MeO}$ & 120552 & and & 10092 & 1320 & $n 2 x$ & 2305 & $a 20$ & $11-10 x x$ & I11: & 41.00 \\
\hline & & & & & & & & & & $11 \cdot 10.28$ & 1222 & acs & 20000 & av & $120 x$ & 1000 & ra14 \\
\hline 1.2 & & & & $(t)$ & 14 & & & & & 12.9 .92 & 1011 & 1580 & 4357 & $a$ & 1.7 .83 & 1136 & 3020 \\
\hline $5.1-92$ & 1180 & $4 \times 12$ & 47455 & ans & $7.12 .9 \pi$ & 1000 & $\operatorname{sen}$ & sous & ans & 1.7 .20 & 2131 & un & seas & a31 & 210.98 & $\infty$ & 41.23 \\
\hline $3-6 \cdot 9$ & 206 & andi2 & seses & 000 & $415-9$ & sos & ans & $\operatorname{son}$ & an & 2.10 .83 & 1006 & 07.16 & 21914 & aso & 29x & 94 & Alat \\
\hline $\operatorname{cis} x$ & BS & ast & singe & 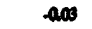 & $2.2 \cdot n$ & 1126 & nes & 20000 & 2005 & 3.98 & s4 & $n, 74$ & 1S19) & ass & & & \\
\hline 7.2 .92 & 230 & an & sens & ant & 2.s. $x$ & 73 & Rst & 31900 & acs & & & & & & & & \\
\hline $27-2$ & $\omega$ & 45 & 30000 & ais & i2s. $n$ & 1236 & 4.13 & 25000 & 000 & 3.12 & & & & & s-1 & & \\
\hline esen & $m$ & $\mathbf{s e n}$ & 2009 & -000 & $1.8 \times 2$ & 106 & 41.74 & so:es & act & 213.92 & 12000 & $\mathbf{1 6 0}$ & 40304 & acs & 21292 & $\infty$ & ser \\
\hline $\operatorname{sen} 2 x$ & $m 7$ & 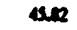 & $32 n$ & ant & $2 \sin x$ & 1030 & 43.17 & 25150 & 000 & 362 & 1130 & 1032 & 10000 & and & 2929 & $\infty$ & Suss \\
\hline $11 \cdot 10-92$ & $m$ & 15.3 & 2357 & $a x$ & $3-5,2$ & 1403 & 120 & 20006 & 000 & $4 x$ & 960 & 462 & 2.1354 & ane & 10092 & 64 & $\operatorname{sen} 1$ \\
\hline 124.92 & $\infty$ & 1306 & $\operatorname{sen} \theta$ & av & 4492 & 504 & 2213 & 20000 & 000 & $\operatorname{sis} 2 x$ & $12 n$ & 4sen & s.7560 & 000 & 11.10 .92 & 1130 & $\omega$ \\
\hline 1.7 .80 & 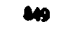 & as18 & $4 \times 002$ & ass & 2692 & $\infty$ & 1199 & $\operatorname{sen}$ & ace & $62 x$ & $122 x$ & Mrn & (67741 & 000 & $12 \cdot 2 x$ & 900 & $\$ 253$ \\
\hline 2980 & $m$ & 45.75 & 2013 & $a 0$ & $6-19$ & 742 & 41.51 & N16S & 201 & $\cos 92$ & 12000 & $M 1$ & 7.19es & -000 & 1.7 .93 & 1146 & 5213 \\
\hline $\operatorname{sen} 0$ & 200 & 42 & 2357 & a.6 & $7.2 x x$ & 740 & 11.36 & 12000 & an & $\cos 2$ & 70 & 100 & asson & 000 & 210.00 & $m$ & 2424 \\
\hline & & & & & 27.9 & n3 & (23)! & 22000 & acs & $\operatorname{sen}$ & 1214 & 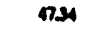 & 3.7m & Q19 & 20.93 & 760 & Sent \\
\hline 21 & & & & & $\log 2$ & 740 & 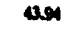 & $1 \mathrm{am}$ & 000 & 10422 & 1327 & 0.15 & 20400 & ast & & & \\
\hline $1-20 x$ & 1030 & 3201 & $\operatorname{sen} 0$ & and & $100-2 x$ & 78 & 4274 & isou & 200 & 11-1ase & 1130 & 60 & 4140 & a17 & 61 & & \\
\hline $2.5-22$ & 1114s & 33.7 & 49\% & 000 & 11.10 .92 & $\infty$ & 1000 & 20012 & +00 & 12092 & 1003 & $\mathrm{Mr}$ & 4906 & 1.46 & $1.22 x$ & & 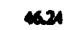 \\
\hline $25-2$ & 1423 & $3 u s 2$ & 4002 & axp & $12+22$ & YI & 41. $\pi$ & $411 n$ & $\infty 00$ & $1.7 .90^{\circ} \mathrm{B}$ & 1124 & Mans & 1006 & IN & 25.92 & 1412 & 461 \\
\hline $\tan 2$ & 74 & 3327 & 2200 & 000 & 1.7 .90 & 50 & ans & coses & 000 & $2,10.90^{\circ}$ & ต3 & 1706 & 1.974 & 210 & 3.5 .92 & 1541 & $\omega \sigma$ \\
\hline sas & 20 & 3232 & 6276 & 000 & 290 & 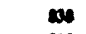 & ance & isen: & aOI & $220^{\circ} \mathrm{B}$ & $\mathbf{2 4}$ & 1750 & 1.000 & 19 & 47.92 & 1043 & 47 \\
\hline $\cos 9$ & 200 & 31.94 & Gnus & $a 00$ & $3-200$ & ns & ax & 1.4900 & 000 & & & & & & $\operatorname{sis} 2$ & 1323 & 4617 \\
\hline 7.2 .44 & 70 & 3227 & 1200 & 191 & & & & & & 41 & & & & & 6292 & 1131 & 4521 \\
\hline $7 \cdot 2 x$ & 125s & 340 & 1.903 & ast & 1.9 & & & & & $129 x$ & 1050 & 20.3 & 1004 & 200 & $630 n$ & 1130 & $\boldsymbol{4 u}$ \\
\hline 27.92 & 245 & 3386 & 30011 & 000 & 7.129 & 1000 & 1897 & N3n & 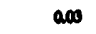 & 1.292 & 1347 & 3200 & 3000 & a.16 & 162 & 97 & $\$ 607$ \\
\hline 9.29 & no & russ & 20065 & 206 & $215-9$ & 201 & 1ess & $3.25 x$ & .036 & $2.13-92$ & 1004 & $\operatorname{sen} \theta$ & S.1000 & Mj & 2092 & 110 & 4734 \\
\hline $100 x$ & $n 3$ & 34.19 & $2 \mathrm{ecoss}$ & and & $22-n$ & 1127 & 2000 & 26037 & ace & 3692 & 1055 & sed & $\sin x$ & ane & 1029 & 1253 & 47.34 \\
\hline $11 \cdot 10.92$ & 200 & 33.9 & 3,3602 & $a x$ & $\operatorname{esc} 9$ & 799 & 2003 & 24397 & and & $\operatorname{sen} 2$ & 1013 & $\operatorname{sen}$ & 3000 & $\cos$ & 11.10 .92 & 1145 & 4721 \\
\hline $12+92$ & $\$ 22$ & 32 & 36197 & an & $12.5-9$ & 1533 & 1284 & 2000 & 006 & $\operatorname{sis} 2 x$ & 123 & 5332 & 65702 & acs & 12.92 & 1130 & $\sin$ \\
\hline 1.7 .93 & 203 & 31.14 & ane & $a$ & 1.99 & rase & 1226 & 41263 & oon & 6.96 & 1150 & 3275 & 72016 & on & 1.7 .90 & $10 \mathrm{~N}$ & 453 \\
\hline $2-293$ & 91 & 3401 & 2003 & act & 2.592 & 1300 & 2.14 & 1.1000 & $\infty$ & $\cos 2 x$ & 1136 & 3214 & 7 ans & 000 & 210.90 & OS & 07.11 \\
\hline 3290 & $m$ & 34 & 25014 & an & 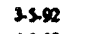 & 1433 & 20 & acoss & 000 & $\cos$ & 48 & 340 & 12000 & 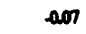 & 249 & 80 & 17.46 \\
\hline & & & & & 402 & 504 & 278 & asens & 000 & $\operatorname{sen}$ & 114 & 3500 & 1046 & an & & & \\
\hline 2.2 & & & & & $2692 \mathrm{~L}$ & 115 & 15.33 & 13,706 & $\mathbf{M j}$ & 1049 & 1300 & $\operatorname{sen}$ & sonor & acs & 21 & & \\
\hline 2162 & 1145 & 3635 & 4050 & 000 & $6-3-92$ & 72 & 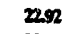 & 25005 & 000 & $11 \cdot 10 x$ & 1104 & seat & 22507 & 200 & $5.1-92$ & $\mathbf{2 0 0}$ & 32.49 \\
\hline 1042 & 246 & $36 n 1$ & $\operatorname{sen} 33$ & all & 7.292 & 200 & 2410 & 4532 & $\mathrm{aOH}$ & 12.92 & 950 & $\$ 20$ & 7,0000 & .0 .10 & 349 & $\boldsymbol{\omega 7}$ & 53.32 \\
\hline $11-10.22$ & 97 & 3624 & 2,2036 & all & 27.92 & n3 & 2018 & 3901 & $\infty$ & 1.7 .83 & 1116 & 31.26 & 207 & a.13 & $02 n$ & 114 & 5252 \\
\hline $124 x$ & 25s & $\boldsymbol{3 \mu}$ & 73153 & al3 & 2092 & 240 & 25 & 1.S19\% & ax & 21000 & 79 & $\sin$ & $\mathbf{5 . 1 3 3 6}$ & an & 63092 & 1090 & 5150 \\
\hline $1.7-9$ & 5 & 3326 & 2000 & $a_{13}$ & $104-92$ & $\mathbf{n s}$ & 2000 & 1098 & $a$ & 3.90 & 200 & 350 & \$5741 & $a 07$ & $4 x$ & 1008 & sen \\
\hline 29.93 & as & 3635 & sion & a.19 & $11-10,2$ & 21 & $2 \times 30$ & 20012 & an & & & & & & $\operatorname{sen} 2$ & 00 & $\sin$ \\
\hline 3.2.9. & 206 & 369 & 120 & ane & 1242 & n1 & $25 \times 4$ & 2720 & as & 47 & & & & & $\cos x$ & $\pi 0$ & $\sin$ \\
\hline & & & & & 1.7 .20 & 900 & $25 x$ & 2000 & a)! & $129 x$ & 1045 & 3524 & 1.0000 & 000 & $11-10.92$ & 794 & 546 \\
\hline swe-1 & & & & & 2020 & $\boldsymbol{w 0}$ & $2 \times 75$ & 1007 & as & 1.920 & 1438 & 30 & 20m: & a.10 & 12992 & $\boldsymbol{m}$ & 32.12 \\
\hline $11-15=$ & 1000 & 303.12 & 100000 & $a 00$ & $3-293$ & a1s & 27.03 & ISHIS & 039 & 2.12 .92 & 1533 & 3) & 30050 & Ask & 1.7 .93 & 1017 & 5256 \\
\hline 12.5 .9 & 141 & 341.35 & 20500 & -910 & & & & & & $36 x$ & 110 & 3724 & $6.4 m$ & a.14 & 2.290 & $\cos$ & sus \\
\hline $12.13-9$ & 1217 & 31.02 & 2352 & $a z$ & $1-108$ & & & & & $4 x$ & 1006 & 360 & 700046 & an & $2-290$ & $\pi 9$ & $\sin \theta$ \\
\hline $12.13 .99^{\circ}$ & 1356 & 21100 & 7.7273 & MS & 1.2492 & IJS1 & 3297 & 22003 & all & 2502 & 120 & 363 & $7.0 \mathrm{n}$ & 000 & & & \\
\hline 1.249 & 140 & 31.43 & 7.2002 & 000 & 25.92 & 1219 & 33.70 & NTS\% & oon & 6.2928 & 1206 & 25.75 & 7006 & 1.11 & & & \\
\hline $2 \sin 2$ & 1243 & 31.30 & $700 \mathrm{~m}$ & an & 25.92 & 1310 & 313s & 10036 & $\infty$ & c1ase & 200 & 3610 & c2014 & 107 & & & \\
\hline $1-5,2$ & 102 & 31.30 & 70006 & 000 & 49 & mI & 3295 & 2.4254 & 000 & $625-92$ & 1000 & H:110 & 10907 & & & & \\
\hline 482 & 73 & 30265 & 20316 & an & $3,6.92$ & 766 & 31.94 & osion & 000 & 6.30 .92 & 1141 & 958 & 3.7434 & 000 & & & \\
\hline $\operatorname{ses} 2$ & 1115 & 3421 & ams & $a 00$ & 6.3 .92 & 78 & 31.87 & ans & and & 268 & $\infty$ & 3700 & 1.2466 & ane & & & \\
\hline 6.2 .92 & 1116 & 30132 & 24053 & and & 7.2 .92 & 76 & 32.17 & 62574 & +000 & $20 x$ & IIss & 379 & ass21 & ane & & & \\
\hline 630.92 & 1046 & 30132 & 22035 & and & 2.7 .92 & 733 & $\sin$ & H1100 & 24 & 10492 & 1310 & 30 & arss & 20 & & & \\
\hline 27.92 & m1 & 30150 & 7.1666 & -a.13 & 2020 & 1236 & 3000 & t.1004 & 000 & $11.10 .2 x$ & 1113 & 3750 & $1 \times 10$ & an & & & \\
\hline 292 & 1200 & 31.53 & 7.1981 & a.13 & 10ses & 200 & $\mathbf{3 k} 11$ & 11001 & and & 129.92 & 90 & 35.65 & 10000 & 000 & & & \\
\hline $10+2 x$ & 49 & 341.25 & $c=$ & $-a .13$ & 11.10 .92 & 96 & 3301 & 1000 & 000 & 1.7 .98 & 1100 & $\mathbf{3} .74$ & anss & and & & & \\
\hline $11 \cdot 10.92$ & ass & $\operatorname{su3.n}$ & ased & .100 & 12492 & $\mathbf{9 0 3}$ & 31.72 & 6.700 & 000 & 2.10 .98 & 75 & 37.75 & .117 & acs & & & \\
\hline $12+x$ & 100 & 24ass & 20512 & 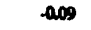 & 1.7 .90 & 92 & $\operatorname{sen}$ & 76054 & 0,00 & $2-200$ & $\$ 4$ & 324 & a.4sm & and & & & \\
\hline 1.7 .80 & 20 & 3418 & 11.186 & and & 2.993 & 900 & 3399 & $20 \mathrm{NI}$ & acs & & & & & & & & \\
\hline 1.15 .8 & 1015 & 30233 & conse & all & $3-9.90$ & 207 & 3478 & 23079 & acs & & & & & & & & \\
\hline 2.293 & 90 & 341.2 & 7000 & a.1 & & & & & & & & & & & & & \\
\hline 3.9 .93 & $\mathbf{6 3}$ & 30011 & 60137 & all & & & & & 3.3 & & & & & & & & \\
\hline
\end{tabular}


errors. Survey or well casing modifications may cause either type of error. Survey errors usually affect accuracy, not precision. Gradual relaxation of the transducer cable causes errors, but they are usually small. Temporary hang-up of a transducer on a pump or other obstruction in the well causes error. Samplers and well maintenance crews frequently remove or adjust pumps; this cause of error is relatively common. Error detection is done by plotting normalized differences between simultaneous water elevations in similar wells. Differences are cause for system calibration recheck.

Errors increase with datalogger range because resolution decreases, as shown in Table 1. Since it is necessary to use a greater range in river stations than in wells, the errors in river measurements are greater in the river data. Errors from topographic survey should be less than $\pm 0.06 \mathrm{ft}$, for most wells.

Transducers produce errors as a result of plugged ports or vent tubes. Water, debris, or a compressed cable cause this type of error. The vent rubes are checked, and a dry desiccant is placed in the vented enclosure to keep air vents clean and dry. Small animals, most likely porcupines, have chewed through three transducer cables during 1993. Larger animals, such as coyotes, have not been able to bite through the cover, however.

If monitor system battery voltage drops too low, directly measured voltages increase. This type error is most likely where solar panels fail to recharge the lead-acid battery, especially during winter time. The pressure transducer and other readings will gradually go too high and will finally be recorded as -99999 when overrange conditions exist.

Errors in the data were detected by listing each file after data download and by field observation. Slipped fittings or observed well pumping or renovation called for error checks.

\subsection{Desiccant and Battery Service}

Battery voltages were measured monthly to detect potential power supply problems without data loss. During December of both 1992 and 1993, battery power proved inadequate on several stations. Low voltages were detected and batteries were replaced without significant data loss. However, the causal problems differed between years. During cold winter weather in 1992, the large, 80-Ah, liquid, lead-acid batteries discharged by parallel connection to the small gel-cell, lead-acid batteries and the sulfate hardened to prevent recharge. The liquid and gel cells should not be connected in parallel unless the solar charger and regulator are capable of voltage output great enough to recharge the liquid cell under low temperatures.

During December of 1993, the systems were operated without the large batteries, and the small gel cells lacked capacity to withstand the prolonged cloudy weather. Solar radiation was inadequate to recharge batteries during November, December, and January. Solar insolation during December 1993 was less than $10 \%$ of typical June radiation. Thus, it was necessary to replace and recharge batteries each week. Solar panel enlargement proved incapable of resolving the problem. Investigation of the power supply issue caused us to decide to do two things in the future: Install 40- to 60-Ah, sealed, lead-acid battery with a 2-yr shelf life, and set voltage regulators to output $14.1 \mathrm{~V}$, with over-voltage supply with temperature drop. This will be done during early 1994. 
The desiccant packs have been replaced when the $30 \%$ indicator button in each datalogger enclosur; began to turn pink or when there was other evidence of humidity. When the packs were dried in the oven at the specified $250^{\circ} \mathrm{F}$, they exploded. After securing new packs, we poked two small tack holes in the clear side of each pack to allow outgassing of the water vapor. We had no further problems. In each enciosure, all entrances except the breather vent were sealed. During cold, wet weather, replacement may be required each month. Desiccant replacement is not usually necessary during warm weather. 


\subsection{Results and Conclusions}

A year's data, from the earliest measured in 1993 up to day 340 (December 6), have been processed and plotted for representative sites in each of the seven areas (see Appendix B). We began by processing and plotting river data to show the potential driving forces for the Columbia River/ aquifer interactions. Then data from selected wells in each area were processed and plotted to demonstrate the relative magnitude of interaction among the wells and between each well and the river. While all of the wells showed long-term change with the river, some wells showed no short-term interaction.

Electrical conductivity and temperature were measured in the river and in some wells. Examples and comparisons of seep conductivity and temperature changes were also prepared.

\subsection{Data and Interpretations}

River water elevation measurements for 1993 are shown in Figure 2. Note that time is displayed in Figure 2 and subsequent figures as day of year to match the database values. The range of fluctuation in Figure 2 is most notable, with the maximum just above $400 \mathrm{ft}$ and the minimum just above $384 \mathrm{ft}$ at the 100-B river station. The single-day fluctuation at 100-B was about $13 \mathrm{ft}$. Also notable in Figure 2 is the attenuation of the daily cycle as the water reaches the 300 Area. The cause of fluctuation in the 300 Area river between days 84 and 115 is evidently influenced by something other than the upstream river (e.g., the influences from McNary forebay and Ice Harbor tailwater).

Figure 3 depicts detail more than Figure 2 for four of the five river stations and more clearly displays the dependence of the downstream stations on the upstream water fluctuation. Again, the attenuation is apparent in the 300 Area. Figure 4 shows a still shorter and different interval for all five river stations. The attenuation in the 300 Area appears to be about $60 \%$ of the amplitude of the $100-B$ station, while all of the other stations demonstrate less than 20\%. Figure 5 shows a nearly constant flow period for 10 days. However, the 300 Area river shows more than a foot of variation. The cause of this variation is the composite influence of the Ice Harbor Dam and McNary Dam releases. In short, the 300 Area is in the McNary forebay and responds to the releases from the Ice Harbor Dam.

Figure 6 shows the fluctuation of three wells and the river in the 300 Area, with the river data from station SWS-1. As expected, the river fluctuates most, and the well farthest from the river fluctuates least. Notably, the maximum river variation depicted is about $10 \mathrm{ft}$, whereas the $100-\mathrm{B}$ river fluctuation for the same period was about $15 \mathrm{ft}$. Figure 7 shows a 3-day interval in which hourly elevations are visible. Clearly, the river appears to drive the variation. Well 2-1 is nearest the river, well 8-1 is farthest from the river, with well 1-2 midway between. It is somewhat surprising that fluctuations in well 2-1 are not larger because the well is within about a hundred feet of the river bank.

Figure 8 shows the 100-F river elevations, with variations from three wells. Measurements started in these wells in late May 1994. There is a clear pattern of related variation among the water levels. Figure 9 shows 10 days of water levels measured hourly. Wells F5-43A and F5-43B differ in that they access different horizons within the aquifer. It is surprising, however, that F5-43B responds to river 
RIVER WATER ELEVATION

$300,100-\mathrm{F}, 100-\mathrm{H}, 100-\mathrm{N}, 100-\mathrm{B}$

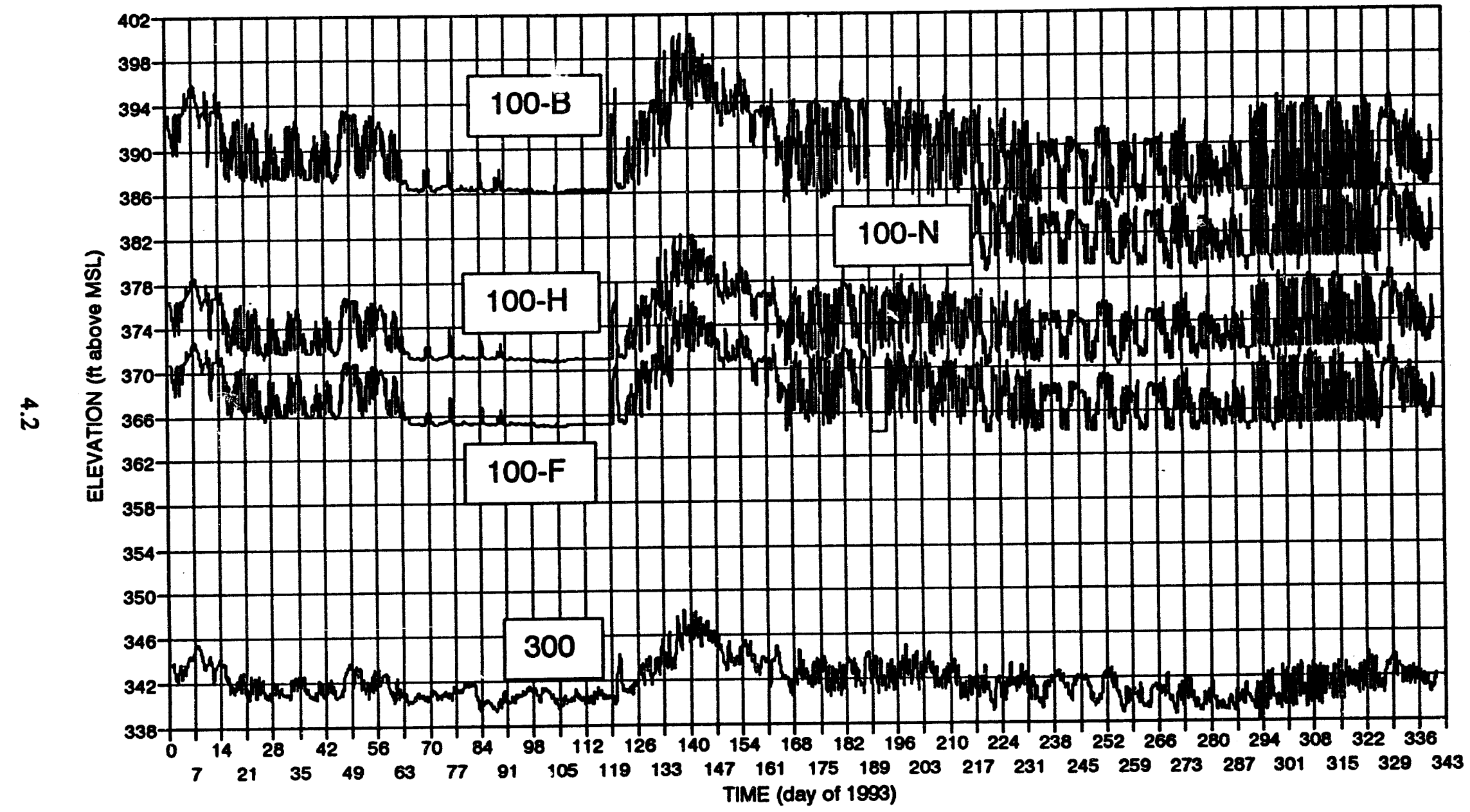

Figure 2. Columbia River Stage at Five Stations 


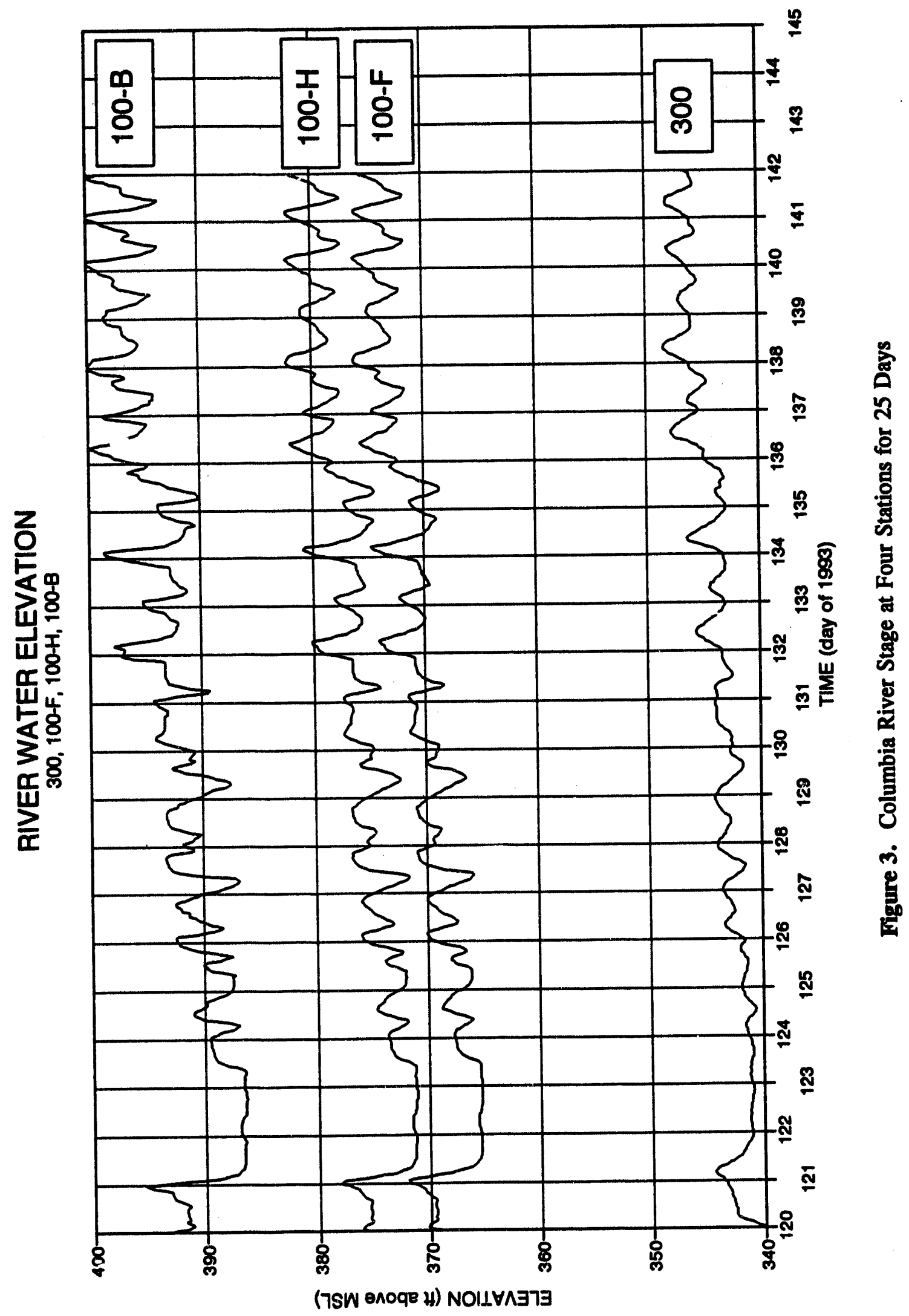


RIVER WATER ELEVATION

$300,100-F, 100-H, 100-N, 100-B$

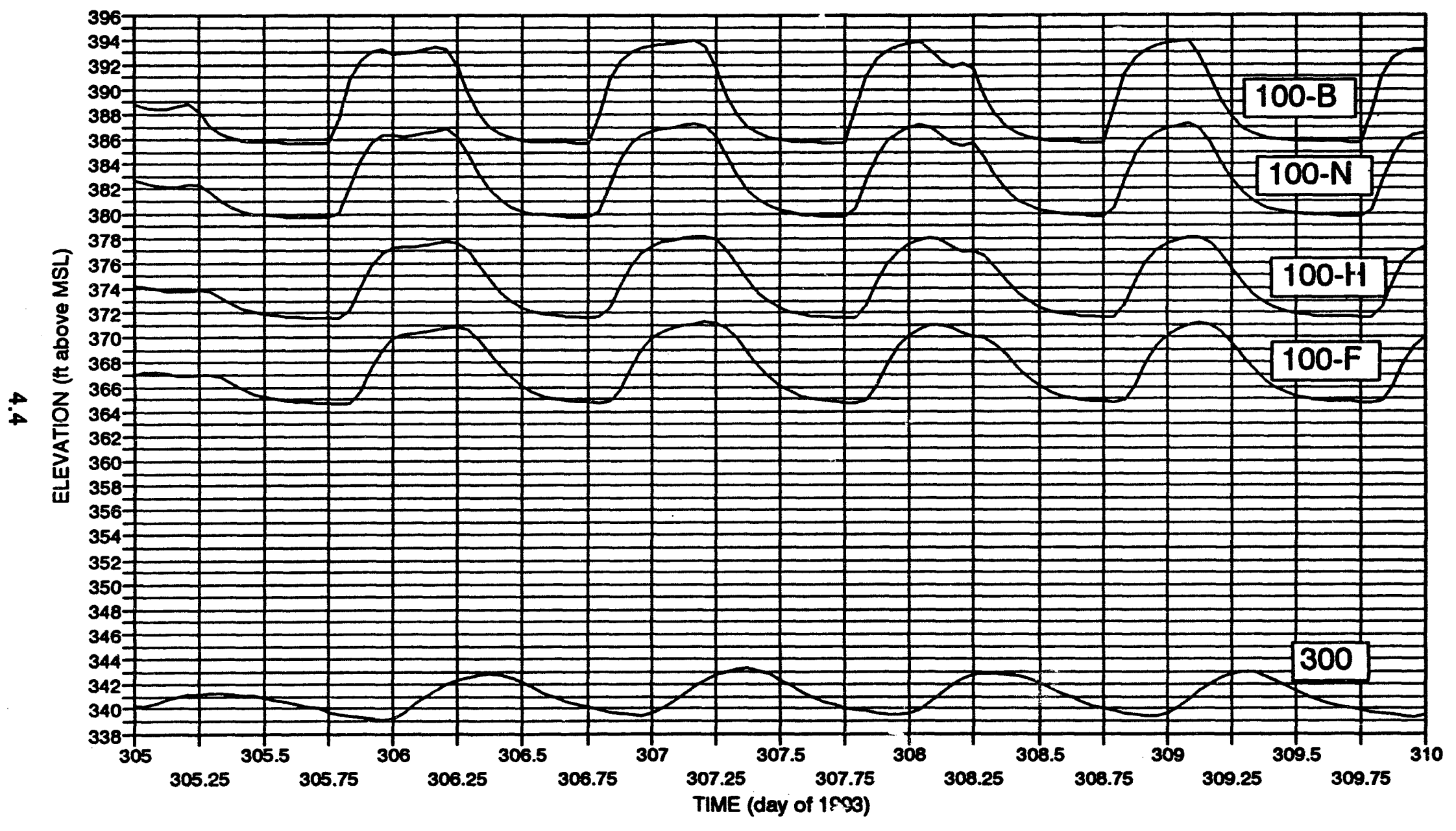

Figure 4. Columbia River Stage at Five Stations for Five Days, Showing Time Lag and Attenuation 
RIVER WATER ELEVATION

$300,100-F, 100-H, 100-B$

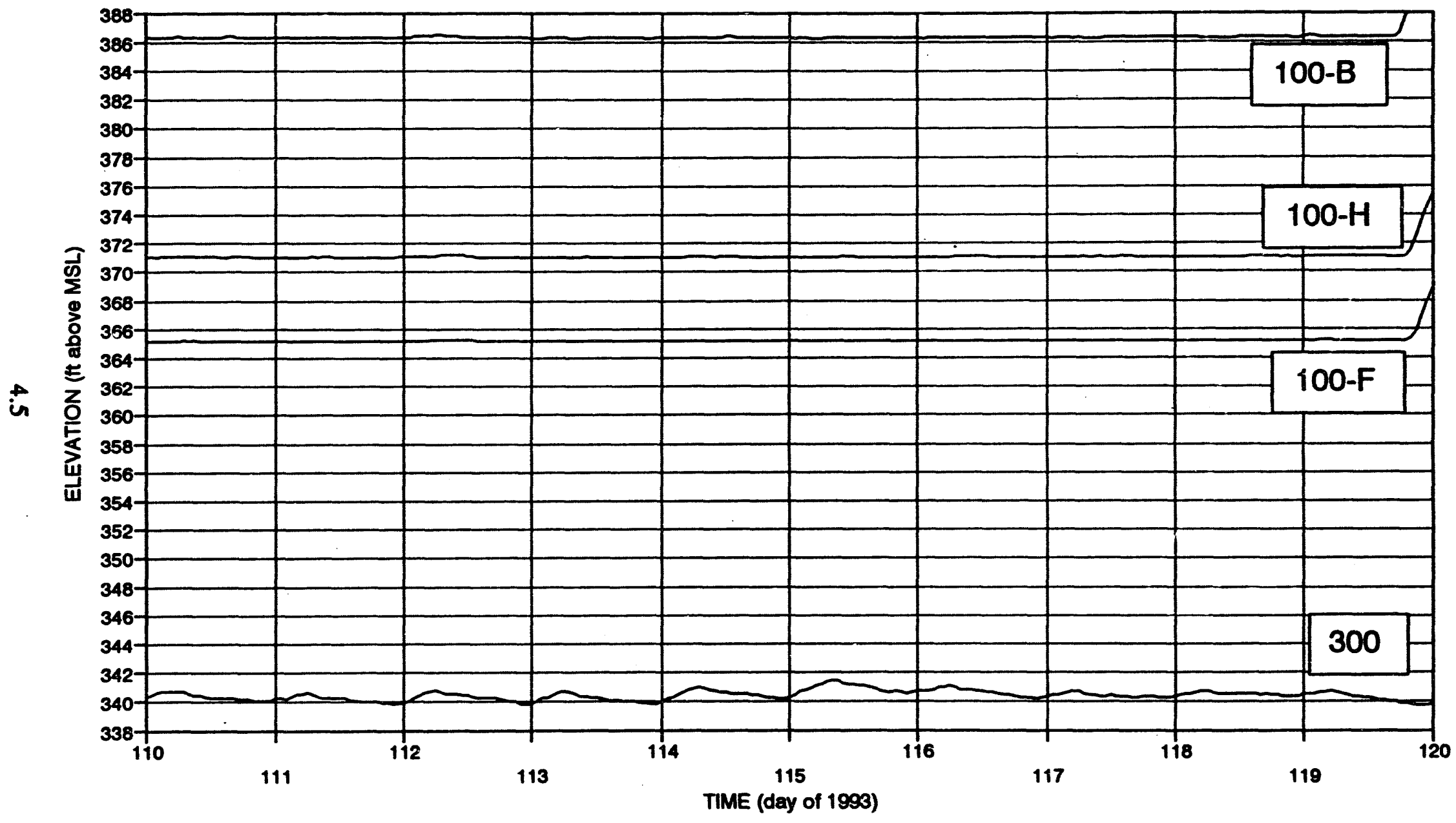

Figure 5. Columbia River Stage at Four Stations During 10 Days of Low Flow 


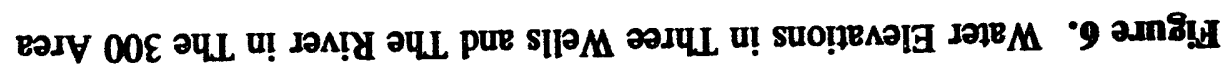

$1-$ SMS $-1-8 * 2-1+1-2+$

(E66। Jo Kep) JWIL

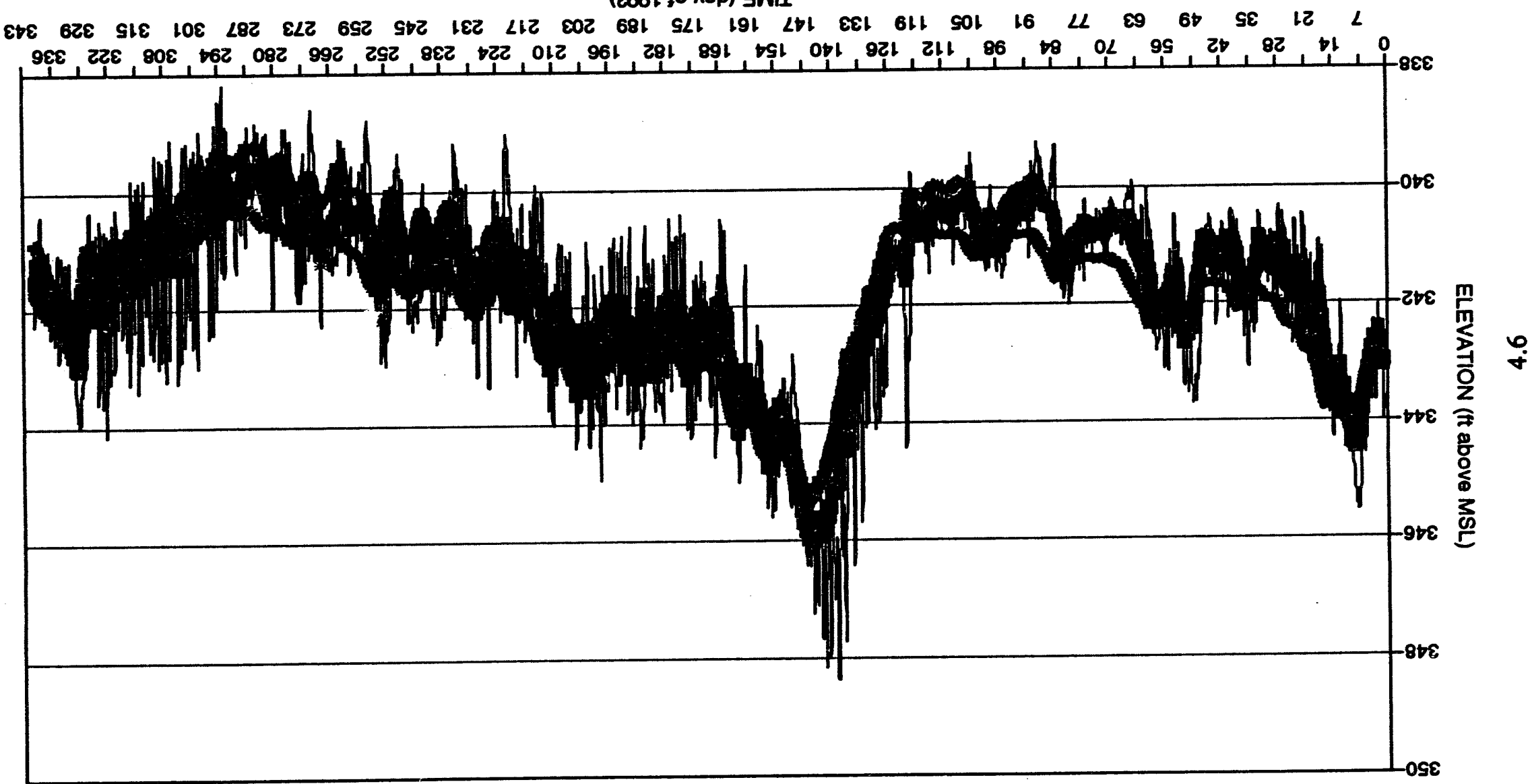




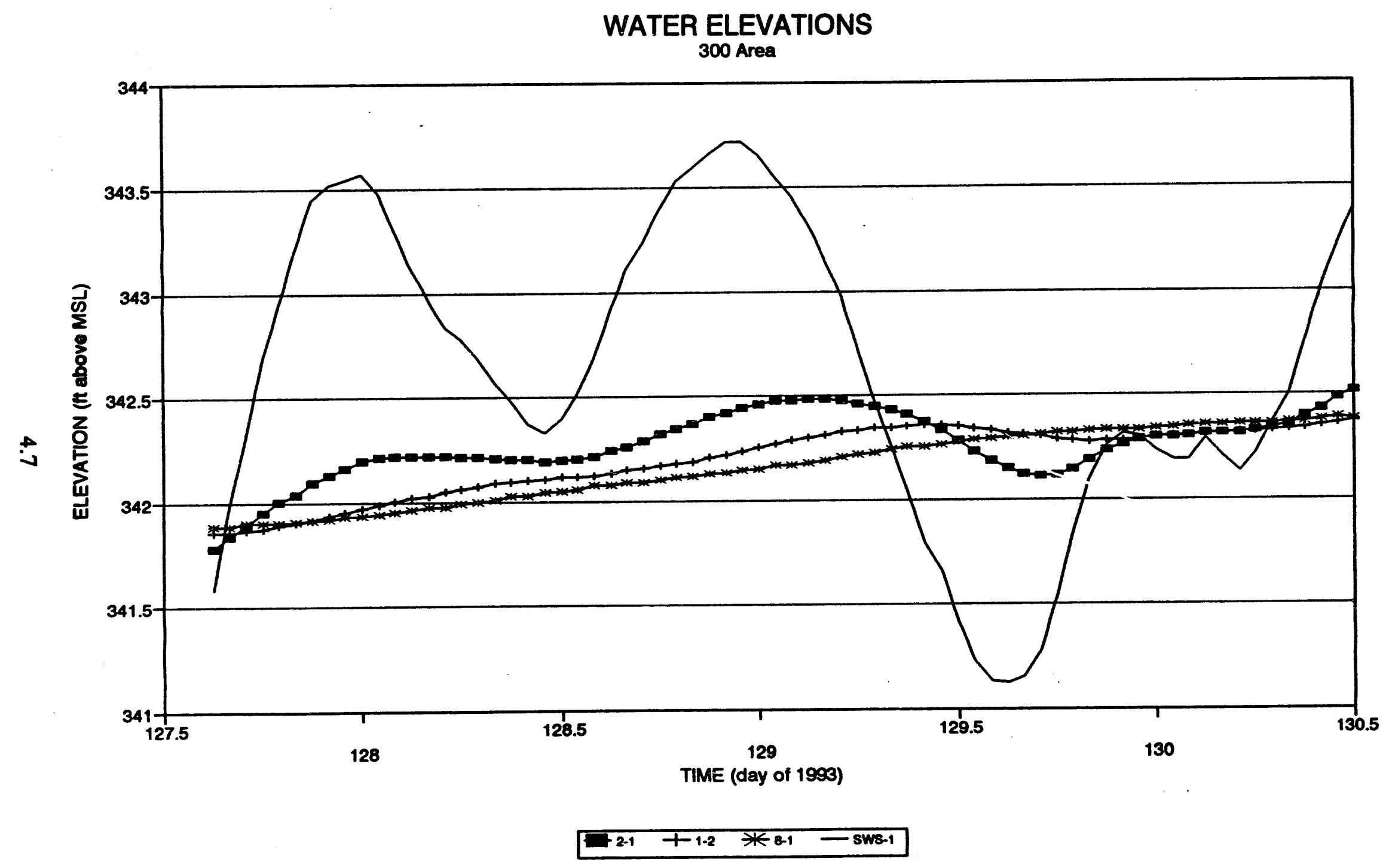

Figure 7. Detailed Variations in Water Elevations in The 300 Area 


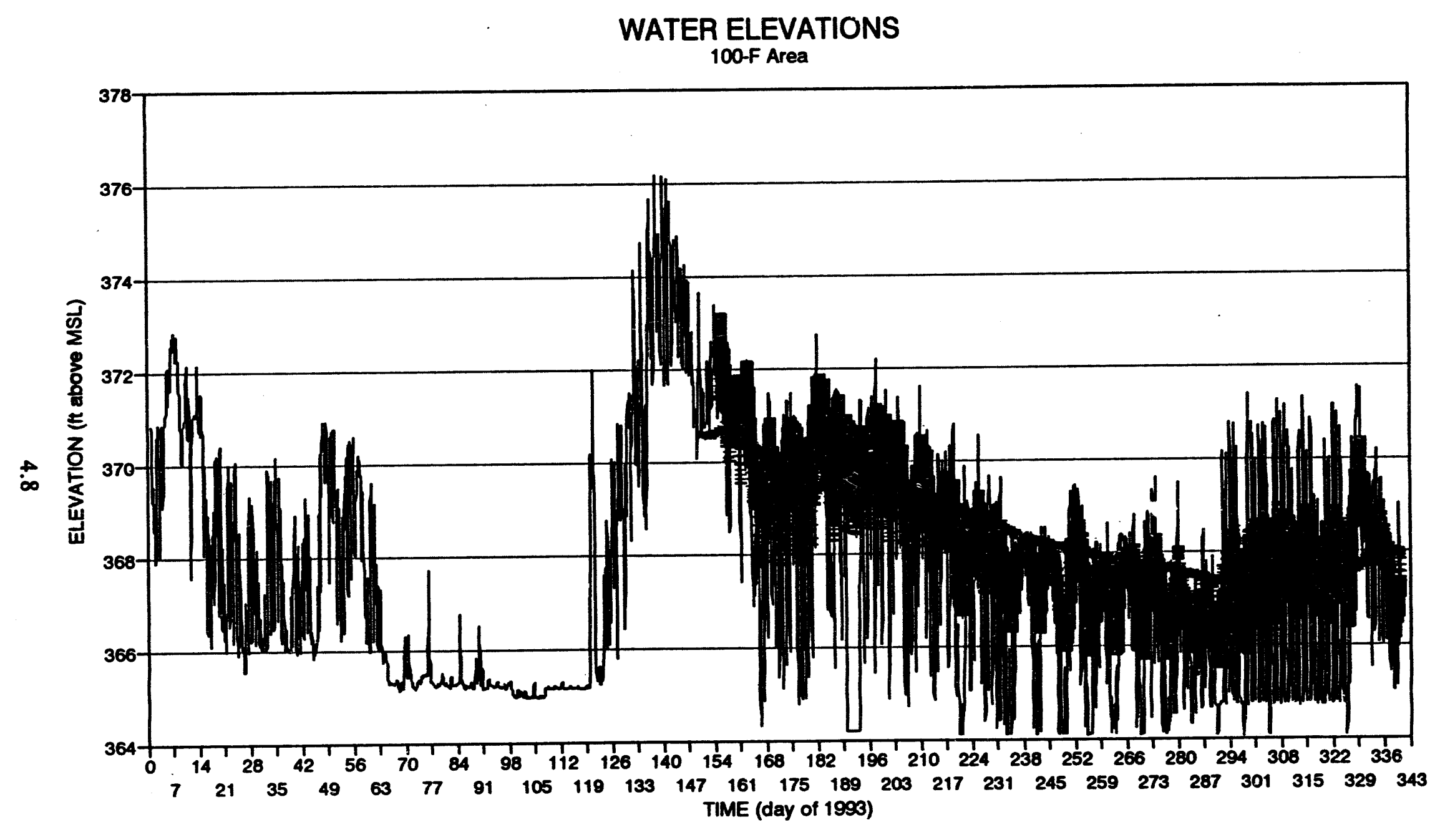

F5-43A + F5-43B * $65-40-F-F i v$

Figure 8. Water Elevations in Three Wells and The River at 100-F Area 


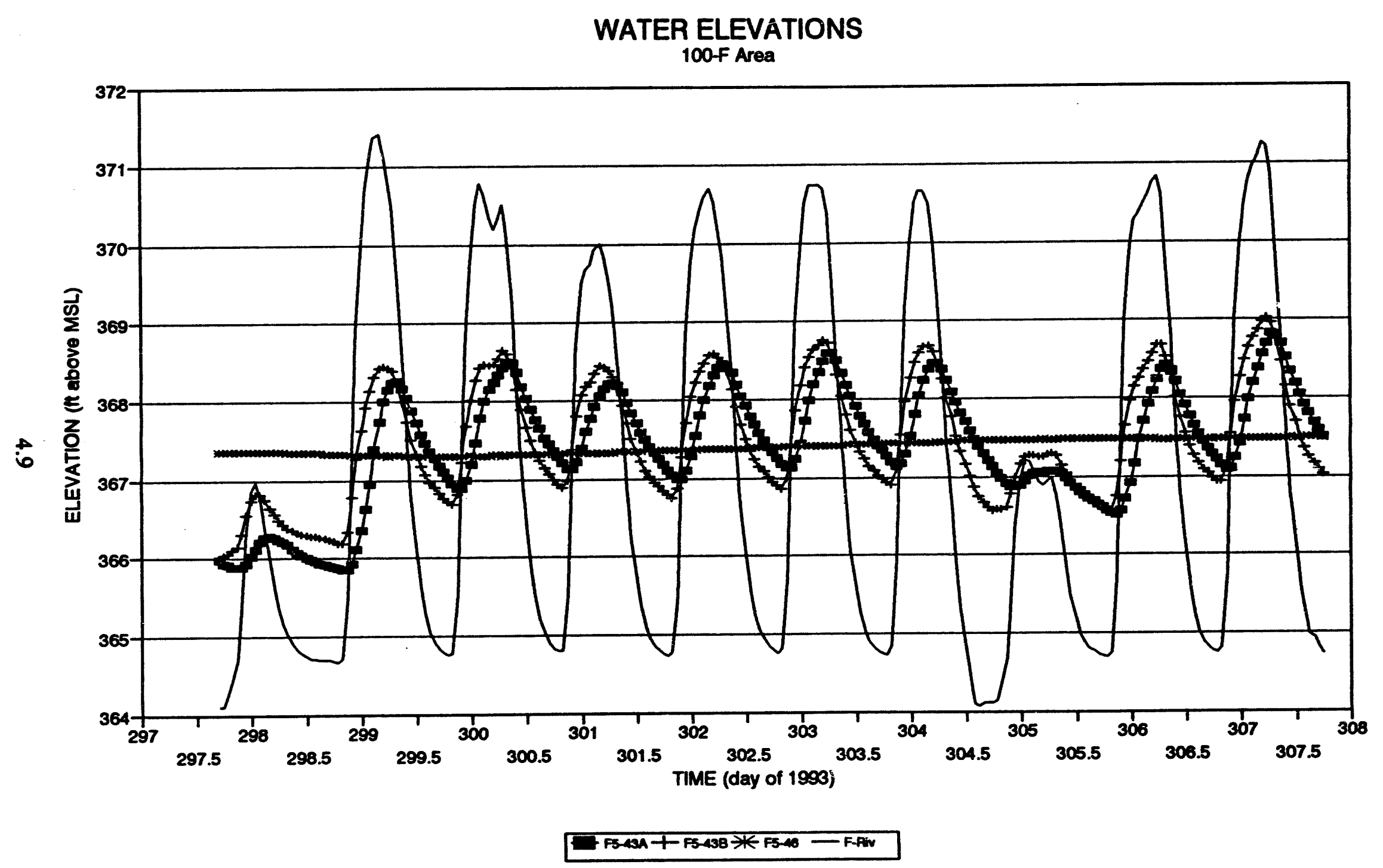

Figure 9. Detailed Variations in Water Elevations in 100-F Area 
influence more rapidly and to a greater extent than F5-43A. Evidently, better hydraulic connection to or partial confinement of the lower phreatic aquifer accounts for the observed behavior in the 100-F Area. While there is long-term influence from the river on well F5-46, there appears to be essentially no influence within the 10-day time interval.

Figure 10 shows water elevations from the river and three wells in the 100-H Area. Here again, the river seems to influence all three wells. Well H4-11 is nearest the river and shows the greatest river influence. However, there appears to be an error in surveyed elevations. This appears to be why well H4-11 elevation is consistently higher than the river water elevation. There may, of course, be another cause for this phenomenon. There may be considerable water flow from up gradient toward the river past well H4-11. While this seems unlikely, it could justify the higher elevation observed in well H4-11. Another possibility is that the 100-H river station survey was incorrect. Resurvey revealed that there was an error of $0.45 \mathrm{ft}$. Also, transducer shift of about $1 \mathrm{ft}$ was logged during tape and datalogger paired tests. The remaining possibility considered was that the top of casing survey for well H4-11 is in error. This has not been resurveyed. Even so, the relation of groundwater elevation to river elevation is unquestionable, in all three wells.

Figure 11 shows a data plot for a 5-day period, with data adjusted to display the relationships between river and groundwater changes. Well H4-11 clearly follows the river, even in fairly minor detail. While wells H3-2A and H3-2C are shown to follow the river in Figure 10, Figure 11 shows very limited response of $\mathrm{H3-2C}$ and no response from H3-2A. This also indicates either better hydraulic contact with the river or the existence of a partial confining layer between the upper and lower levels of the aquifer, as was the case in the 100-F area in well F5-43B.

Figure 12 shows a composite of wells in the 100-D and $-\mathrm{K}$ Areas with the 100-B river data superimposed. The 100-N river station was not installed until about day 217. Again, there appears to be a long-term trend for most wells to follow river fluctuation, with a greatly attenuated amplitude. Well D5-20 shows significant short-term fluctuation, with only token response from well K32A. Well D5-15 data remains unexplainable, though transducer error was a factor, and the transducer was replaced. Still, well behavior seemed independent of river fluctuation. Figure 13 demonstrates the short-term independence between the 100-D and $-\mathrm{K}$ Area wells and the river fluctuation. While Figure 4 showed the common pattern of fluctuation among all four 100-Area river stations, none of the 100-D or $-\mathrm{K}$ wells varied with this short-term pattern. Only well D5-20 shows cyclic response as short as 7 days.

Figure 14 shows water elevations in four wells in the 100-B Area, along with that in the river. Water levels in all four wells seem to vary with that in the river. Although all well water levels shown appear to be above the river level, it is helpful to recall from Figure 2 that 100-B river water elevations were more than $400 \mathrm{ft}$ above MSL for a short period preceding the data interval shown. Figure 15 shows how well the well and river water elevations coincide. It would be more comfortable to explain the fluctuations in Figure 15 if well B2-12 were exactly $2 \mathrm{ft}$ lower. However, well B2-12 is right next to well B3-47, and the tops of casings appear to be about the same, which survey has confirmed. Perhaps tape records were in error; but that is unlikely, based on seven separate sets of paired measurements without significant computed error. We would like to adjust the river level up, but the data from well B3-47 confirms a good match between it and the river. The only remaining explanation we have considered is the possibility that well B2-12 is upstream from the river in a close-coupled 


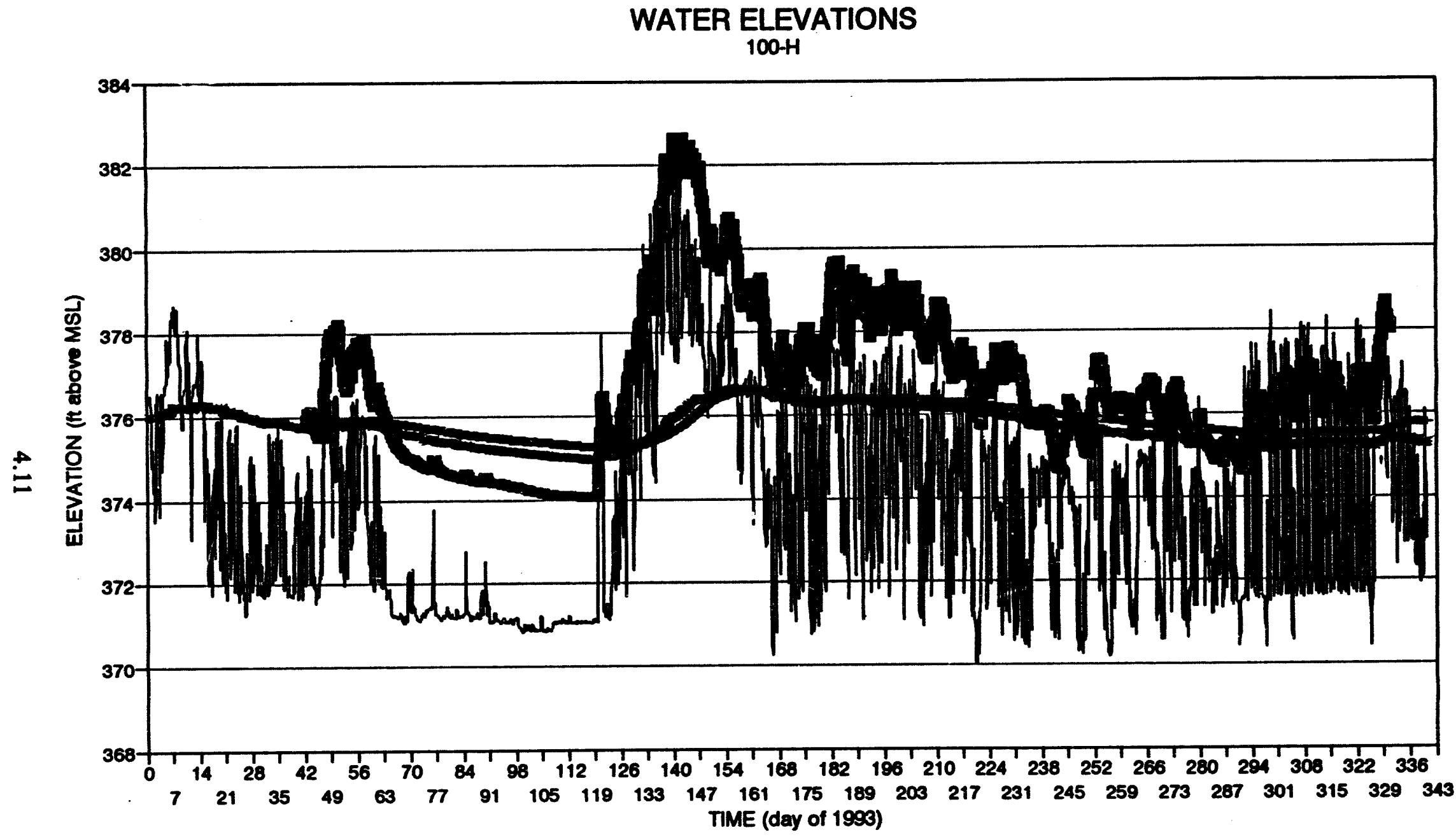

HA-11 $+\mathrm{H}-2 \mathrm{~A} \rightarrow \mathrm{H}-\mathrm{H}-2 \mathrm{C}=\mathrm{H}$-Fiv

Figure 10. Water Elevations in Three Wells and The River in 100-H Area 


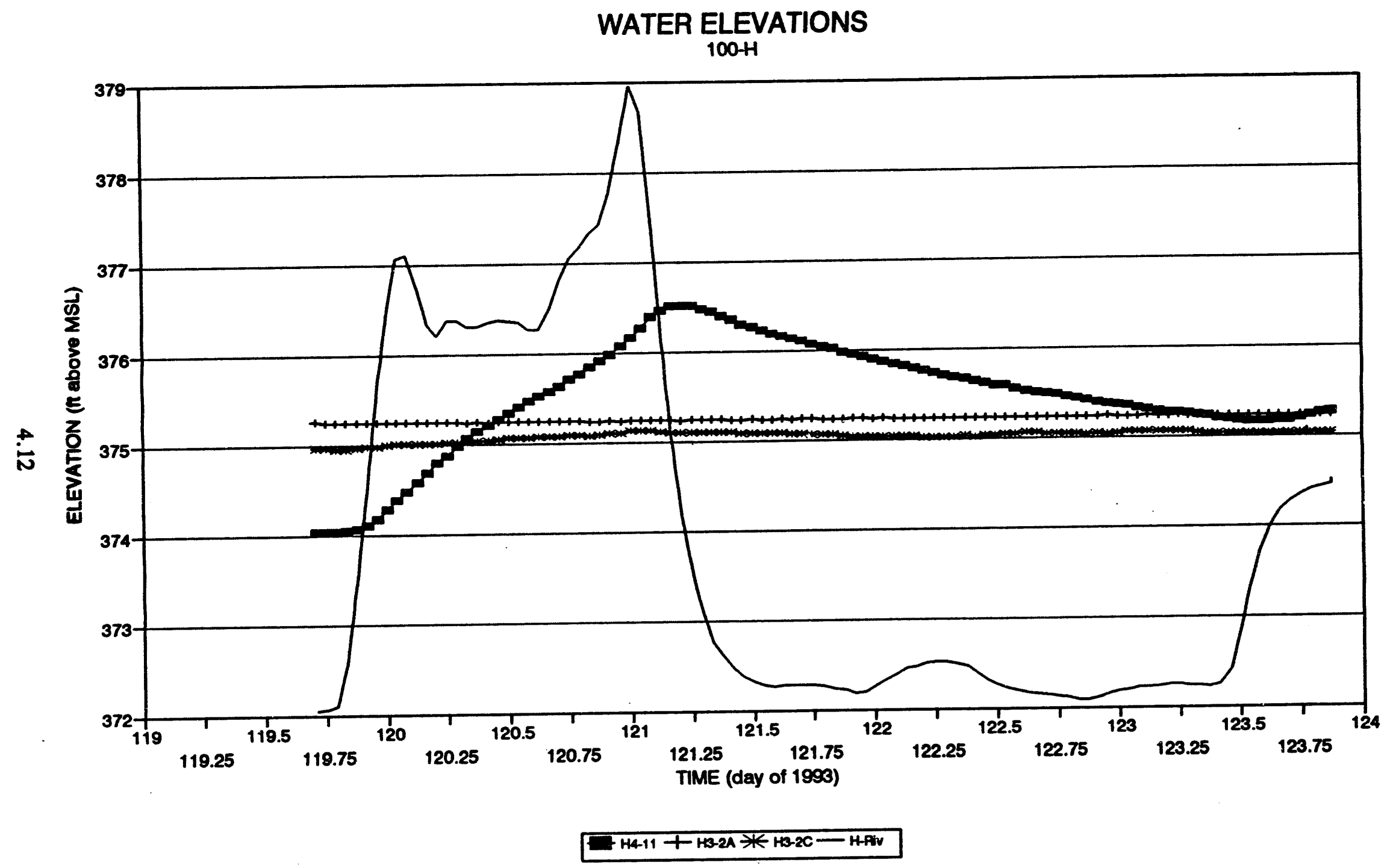

Figure 11. Detailed Variations in Water Elevations in 100-H Area 


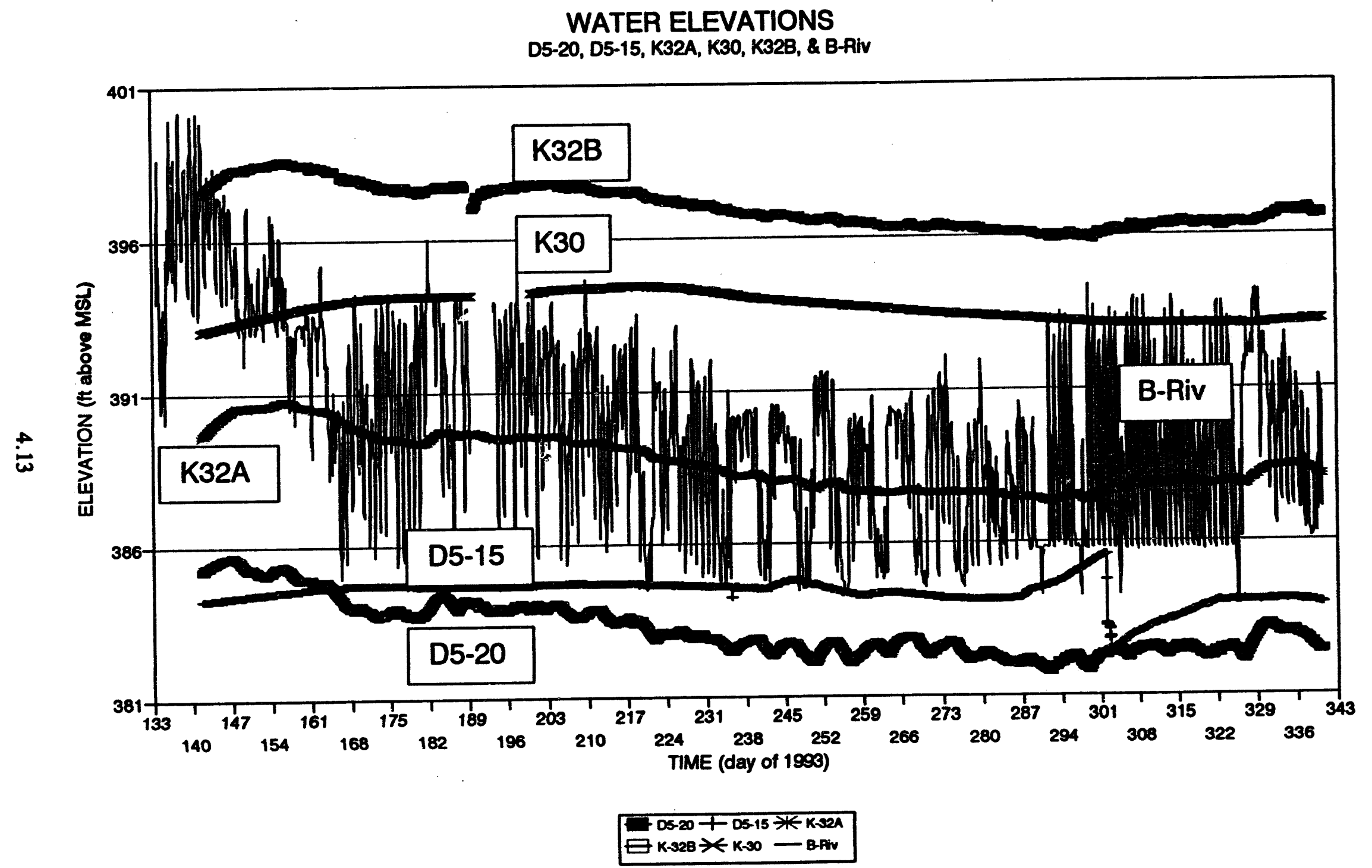

Figure 12. Water Elevations in 100-D and 100-K Wells and The 100-B River 


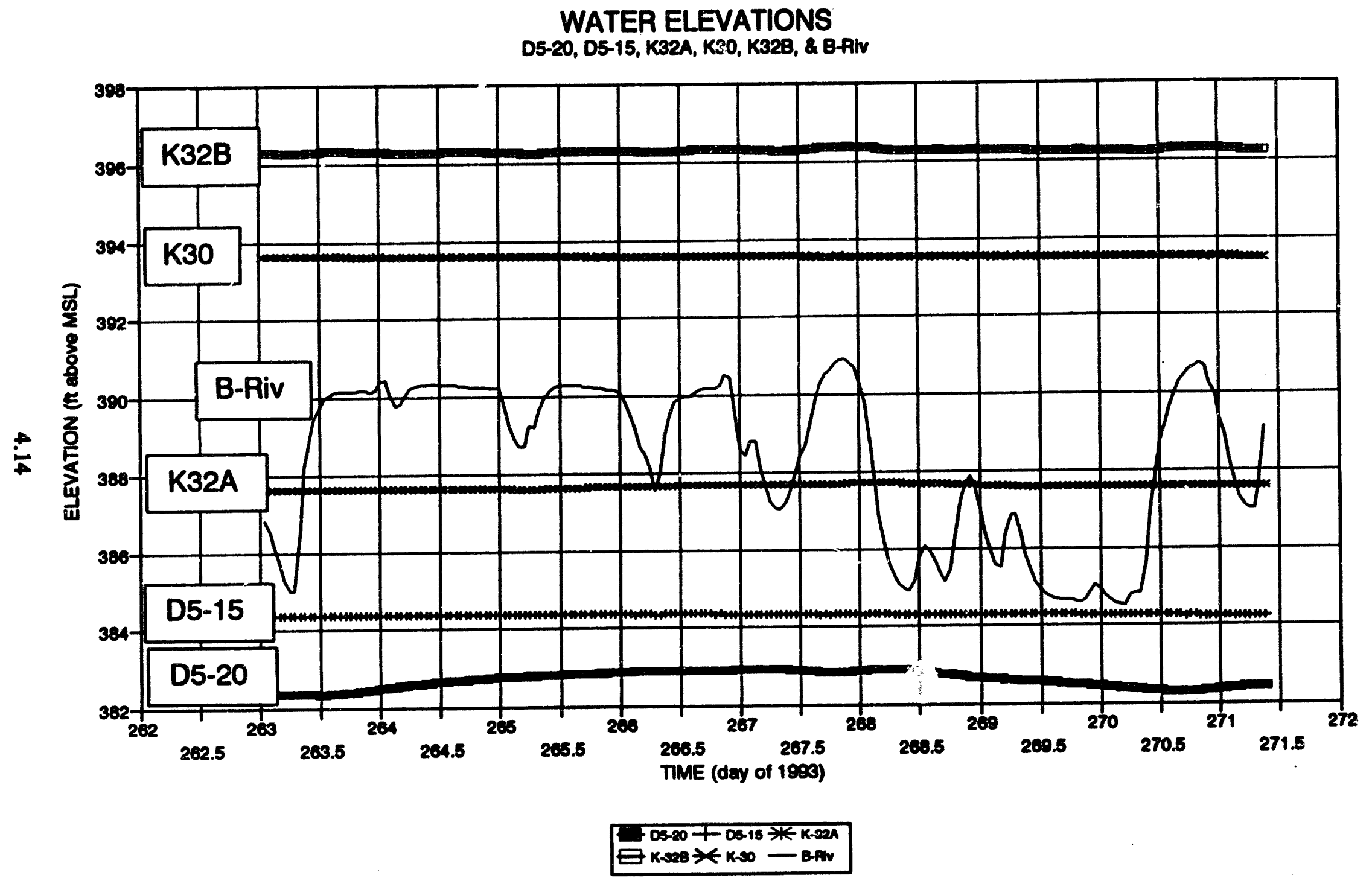

Figure 13. Detailed Variations of 100-D and 100-K Wells and The 100-B River 


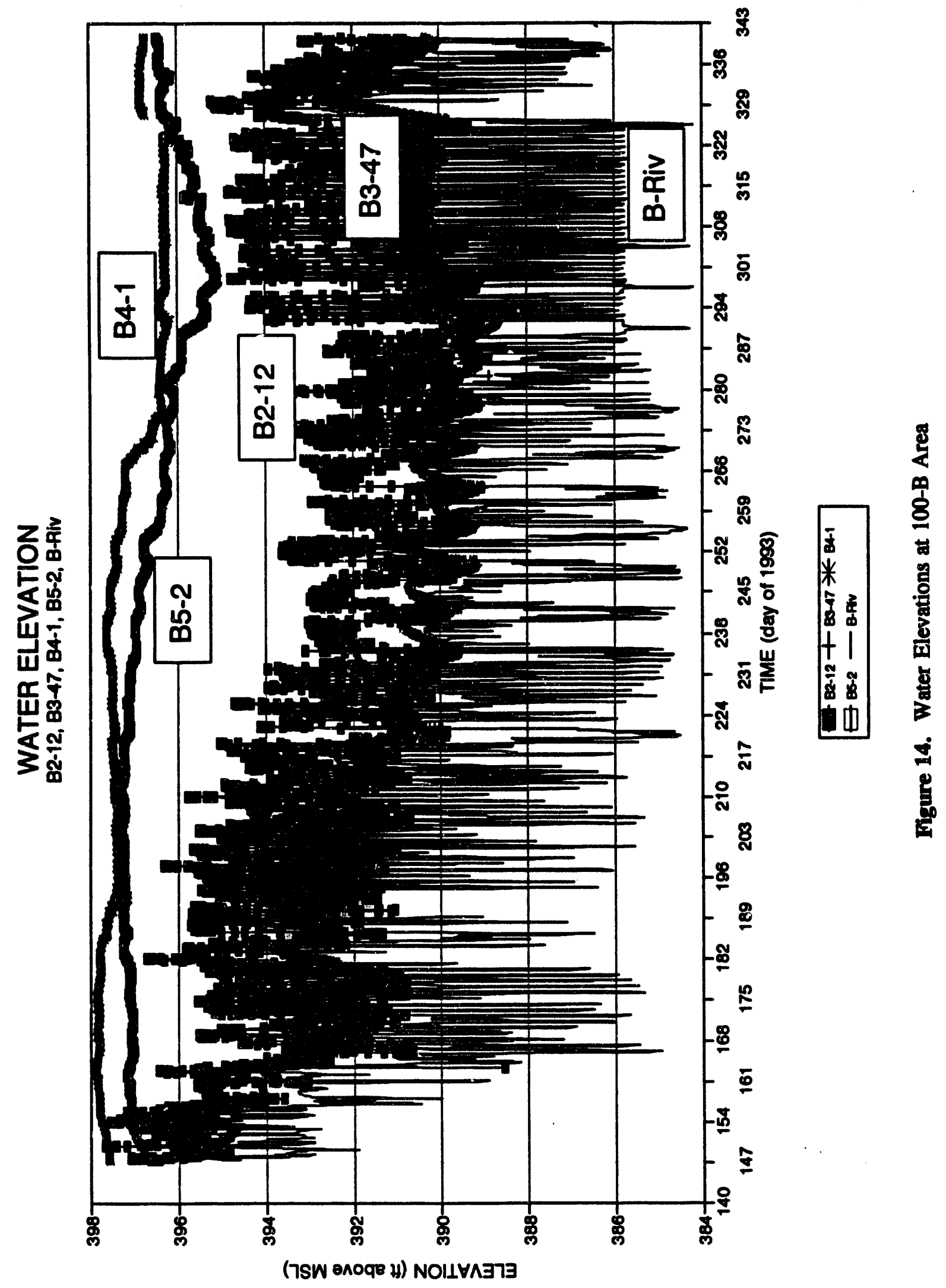




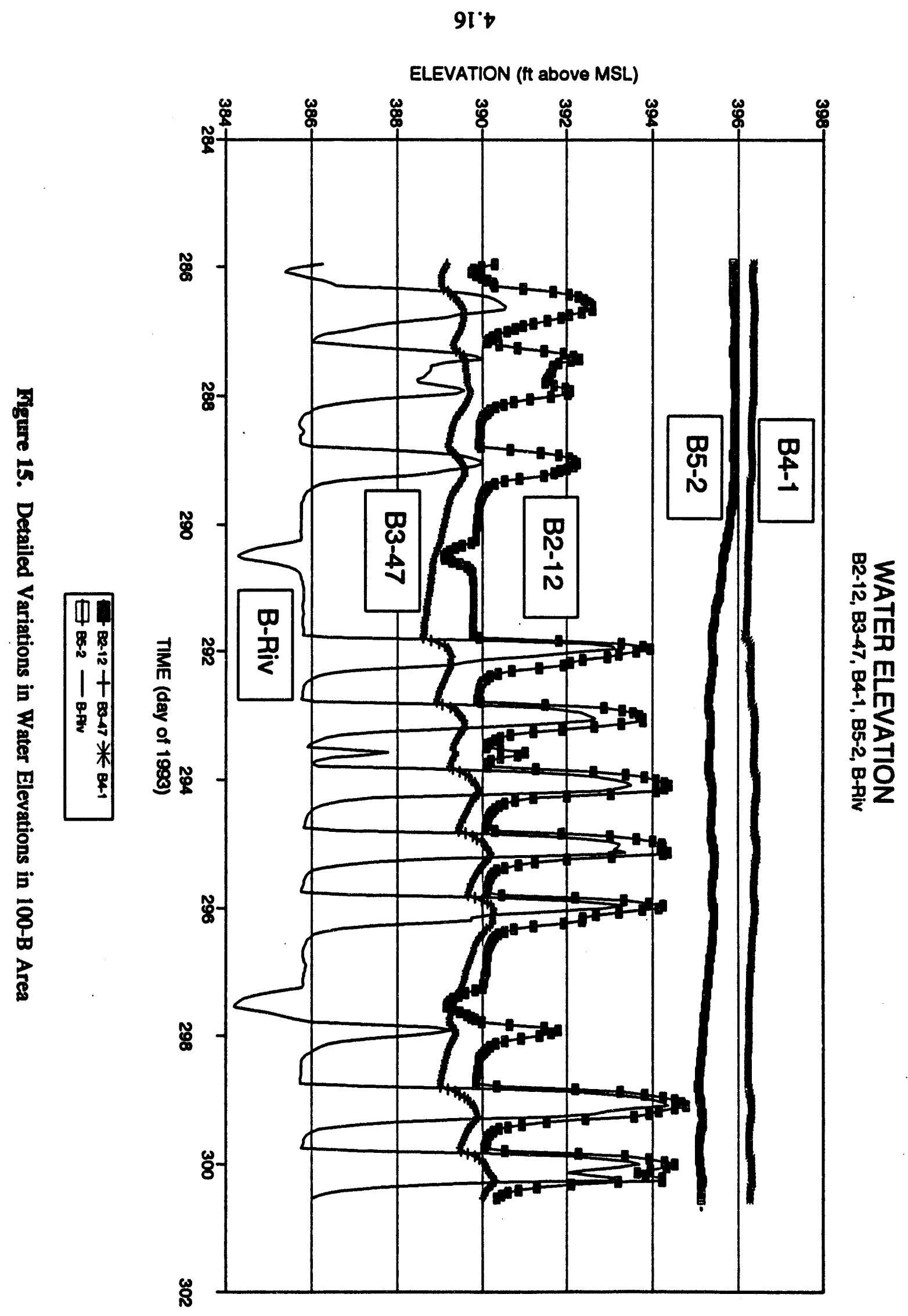


aquifer channel. This close-couple status is apparent from the detail with which the river level is duplicated in well B2-12. Wells B4-1 and B5-2 are distant from the river and are expected to have slow response, with $\mathrm{B} 4-1$ being the more distant.

Electrical conductivity (EC) was measured in the 100-H Area by sensors located in the river, well H4-11, and two nearby river-bank seeps, S-152-2 and S-153-1. Figure 16 shows well H4-11 EC as relatively constant at $0.35 \mathrm{mS}$. The river EC is even more constant at about $0.1 \mathrm{mS}$. While neither of these values is temperature compensated during the interval shown, their relative magnitude remains unaltered after compensation. Temperature compensation and individual calibration followed this interval when the transducer had to be replaced because a porcupine destroyed it. The EC at seep S-153-1 varied from the river value up toward the well value when the river flow dropped below the elevation of the seep, only to decline again as the river water covered the transducer. Just after day 180 , the transducer reading dropped to zero for several hours. The same phenomenon occurred on day 184 and again about day 186. This drop to zero was caused by a reduction in bank storage and failure of the seep to yield drainage water. It is likely that the seep EC never would rise to the level of well H4-11 because bank storage represents a zone of river intrusion and influence. The evidence for this conclusion is shown at about day 172, where the river is not directly influencing the conductivity of the seep and yet the seep EC does not rise. On the other hand, well H4-11 clearly shows the influence of intruding river water, especially when the river level fluctuates strongly above the $375-\mathrm{ft}$ elevation.

Figure 17 shows temperature compensated EC for the stations displayed in Figure 16 and for seep S-152-2 at the outfall. Here, the seep EC does exceed that of well H4-11 for a short interval when the river drops below the 375-ft elevation. Again, seep S-153-1 ran low on water when the river dropped below the 375-ft elevation. At no time did seep S-152-2 appear dry. Instead, it seeped up through the cracks in the concrete outfall apron and formed a significant stream that flowed off the apron and into the river.

Figure 18 shows some water temperatures measured by the EC probes. The large fluctuations near day 182 resulted from drying of the seep, leaving the transducer exposed to the atmosphere. The low temperatures shown near day 325 also represent periods of transducer exposure to air because of seep dry-down. The temperature in well H4-11 remained nearly constant near $20^{\circ} \mathrm{C}$, while the river varied from about 4 to $22^{\circ} \mathrm{C}$. With these relative temperatures, river water would be expected to intrude beneath the groundwater during cold periods and over the top during warm periods. It would be useful to find a long, screened section of well near the river where EC could be measured to distinguish river water intrusion level. 


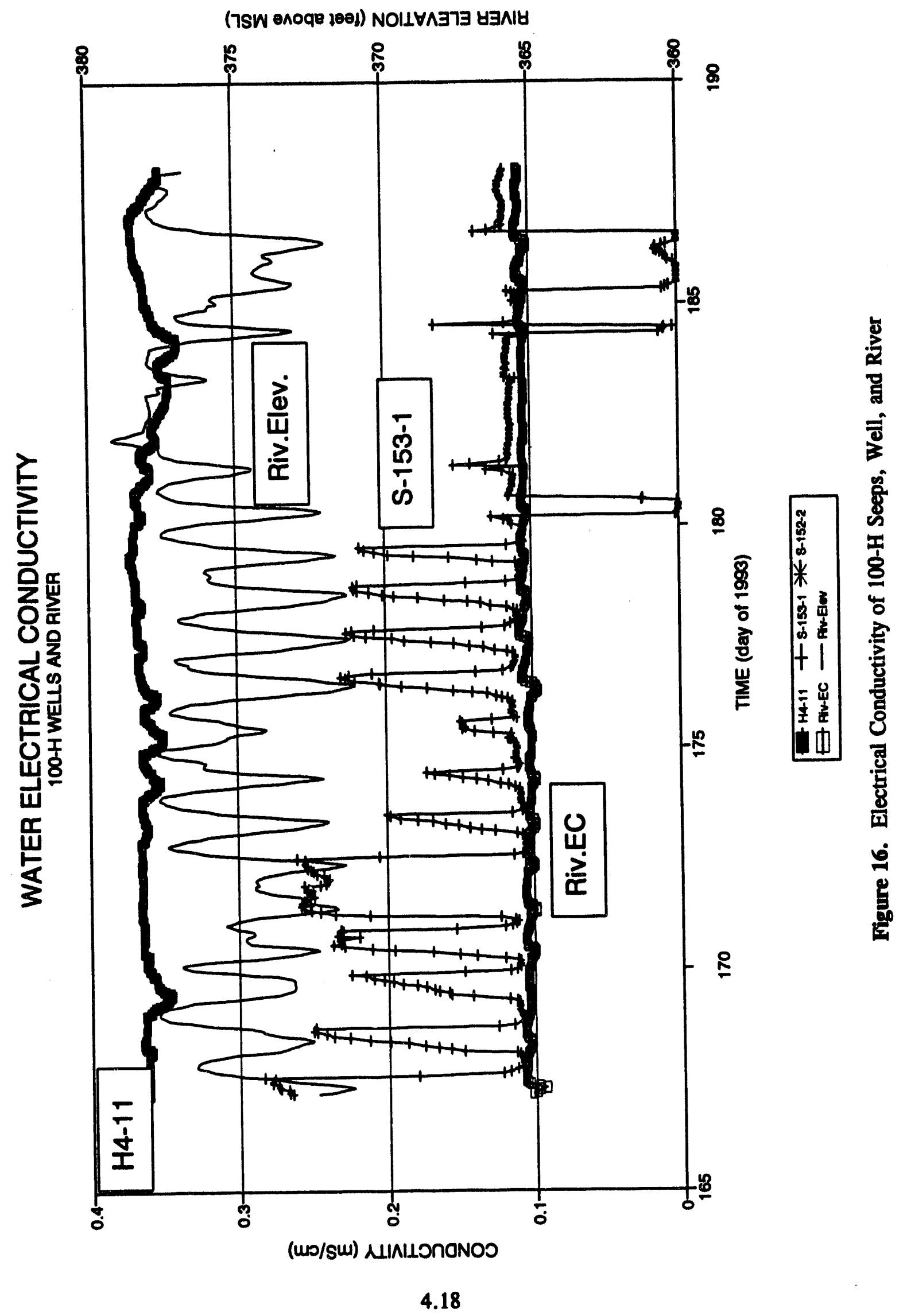




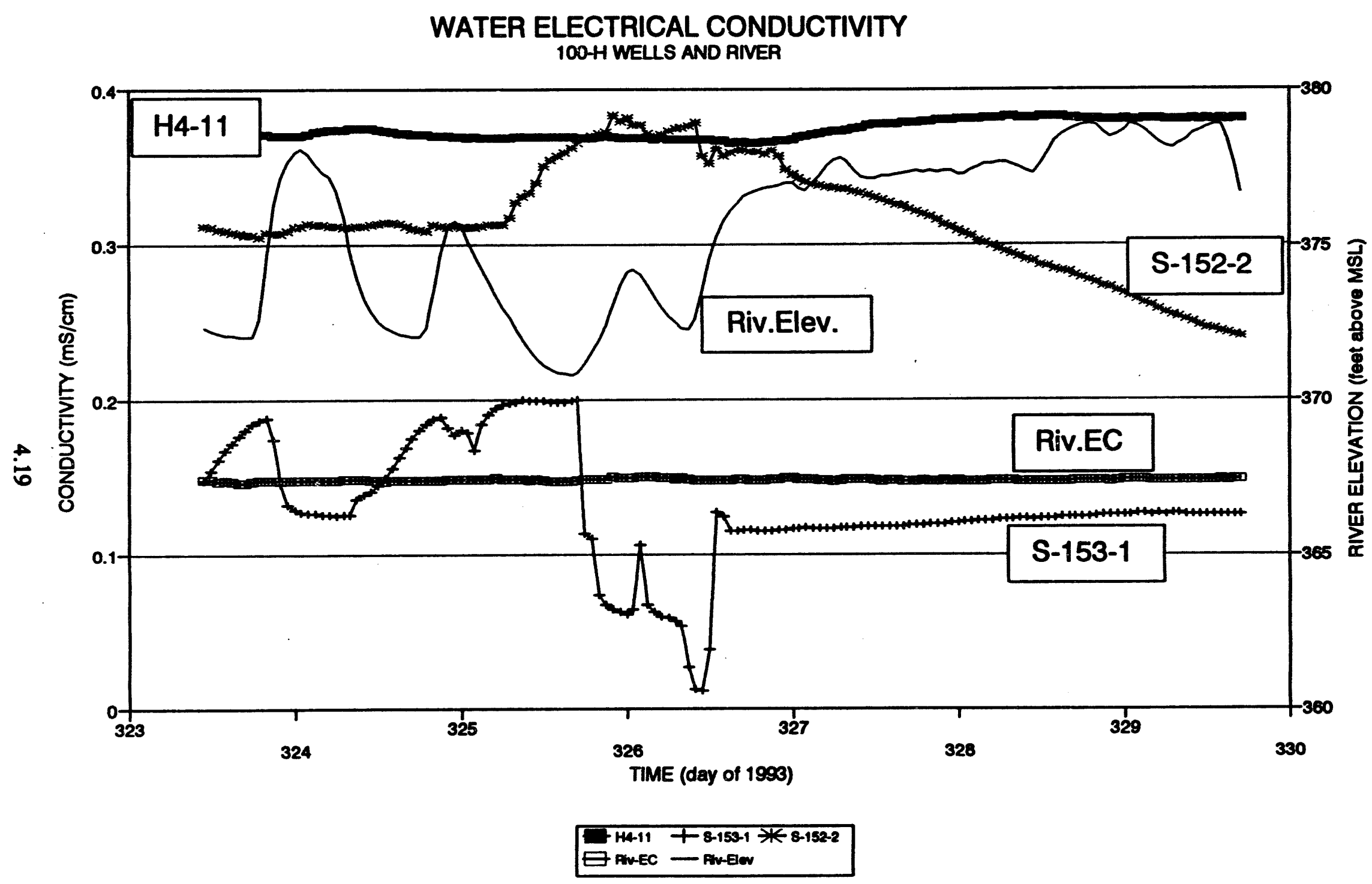

Figure 17. Detailed Variation of Electrical Conductivity at 100-H Area 


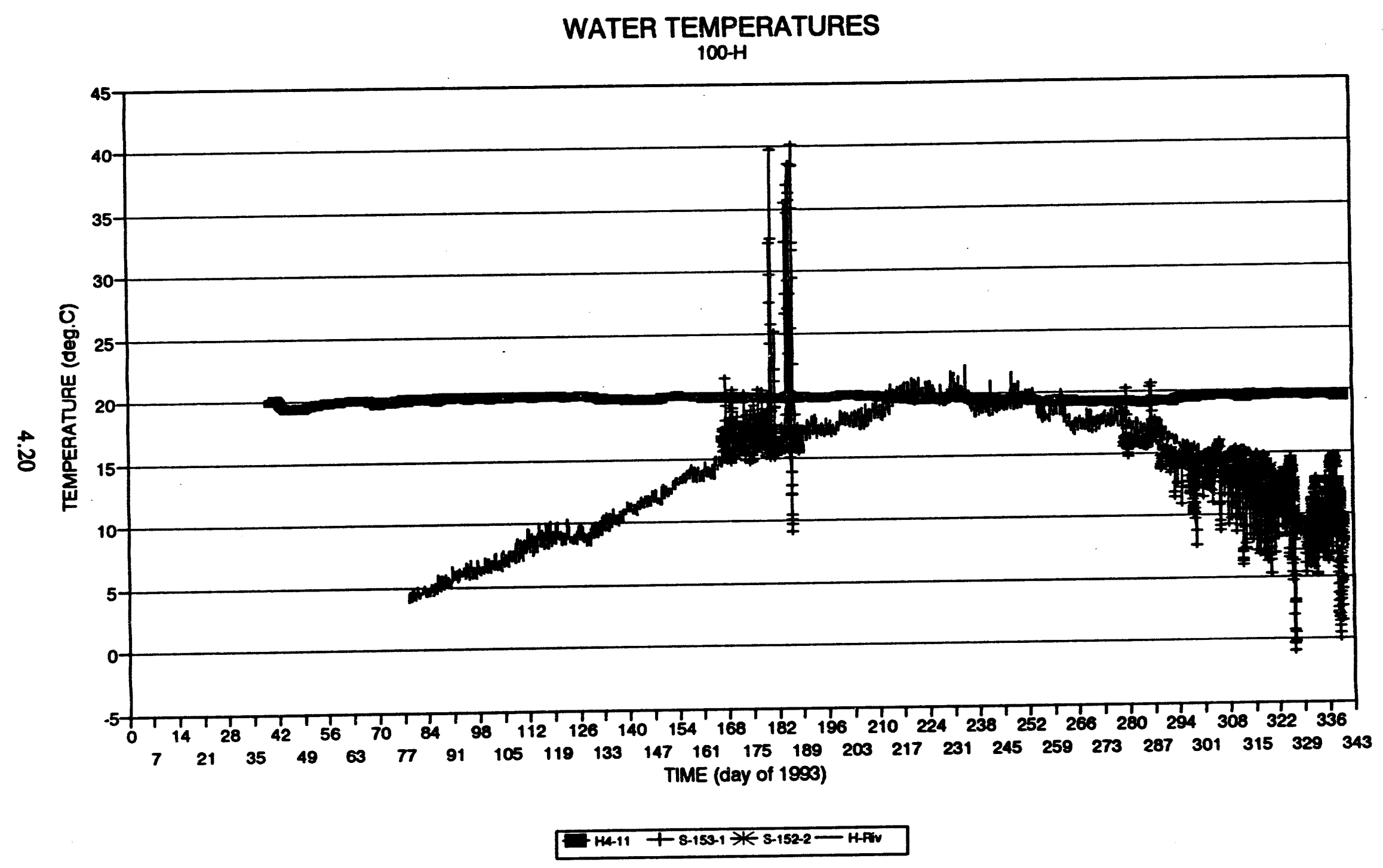

Figure 18. Thermal Variations of River and Groundwater at 100-H Area 


\subsection{Conclusions}

Emphasis and equipment shifted from the 300-FF-5 Operable Unit to the 100 Aggregate Area Operable Unit during spring 1993. Monitors removed from the 300 Area were installed in $100-D,-N$, and $-\mathrm{K}$ Areas and added to the number in the $-\mathrm{F},-\mathrm{H}$, and $-\mathrm{B}$ Areas. Eleven wells and one river station are still monitored in the 300-FF-5 boundary while the others were spread about evenly among the 100 Areas. The monitor network currently consists of 44 radiotransceivers and 42 automatic dataloggers, with one station in each area, except 100-N, serving two wells.

Electrical conductivity and temperature, measured at well, seep, and river locations, showed the influence of intruding river water in both seep and well water. Measurement frequency remained at 1 -hr intervals.

Elevation accuracy is believed to be within $\pm 0.1 \mathrm{ft}$. Topographic surveys, instrument calibrations, and steel tape measurements all contribute error. Short-term network precision appeared to be within $\pm 0.02 \mathrm{ft}$. Steel tape measurements were read within $\pm 0.01 \mathrm{ft}$. Survey error varied with distance from the reference, but was probably less than $\pm 0.07 \mathrm{ft}$. Periodic tape and datalogger tests helped ensure precision and accuracy by including the entire measurement system in the tests. Accuracy of survey errors or ground shift approached $0.5 \mathrm{ft}$ at SWS-1 and $100-\mathrm{H}$ river stations.

Examples of conductivity, temperature, and water levels were presented for each area monitored. River water levels at all five river stations were shown to fluctuate together, with a phase shift and amplitude change to adjust for river mile and channel cross section. Only in the 300 Area was the river amplitude attenuated significantly because of the McNary Dam forebay. Ice Harbor influence on the water level in the 300 Area is apparent when water elevations drop to $340 \mathrm{ft}$ above MSL.

Some equipment problems were reported, such as transducer drift and battery discharge. Transducer drift was resolved by replacement or compensated by periodic manual measurements using steel tape coupled with datalogger readings. Questionable data were tested by comparison with similar stations. For example, 100-H and 100-F river stations were compared by subtracting the mean and dividing by the standard deviation. This normalized the data about a common zero. It did not correct for data divergence due to time lag. Where data drift was apparent, linear adjustment was used from the last known correct point to the measured divergent point. Long-term drift of transducers was accommodated in this manner. Key factors in data reliability were in situ calibration of pressure transducers, periodic steel tape and datalogger paired readings, difference tests of data, and visual data checking. Solar radiation at Hanford was inadequate to recharge batteries during November, December, and January. Experience demonstrated the necessity to use batteries with at least 40-Ah capacity to operate the network remote stations reliably during this period.

Water levels, temperatures, and electrical conductivity measured by the automatic monitor network provide an initial database with which to calibrate models and from which to infer ground and river water interactions for site characterization and remediation activities. 


\subsection{Bibliography}

Campbell, M. D., R. Schalla, and D. R. Newcomer. 1991. Accuracy and Cost Effectiveness of Manual and Automated Water-Level Monitoring Technology. PNL-7566, Pacific Northwest Laboratory, Richland, Washington.

Campbell, M. D., and D. R. Newcomer. 1992. Automatic Measurement of Water Levels Within the 300-FF-5 Boundary. PNL-7874, Pacific Northwest Laboratory, Richland, Washington.

EPA (U.S. Environmental Protection Agency). 1986. Resource Conservation and Recovery Act (RCRA) Ground-Water Monitoring Technical Enforcement Guidance Document. OSWER-9950.:, Washington, D.C.

Ferris, J. G. 1952. Cyclic Fluctuations of Water Level as a Basis for Determining Aquifer Transmissibility. United States Department of the Interior, Geologic Survey, Water Resources Division, Ground Water Branch, Ground-Water Hydraulics Section, Washington, D.C. Contribution No. 1, April 1952.

Lindberg, J. W., and F. W. Bond. 1979. Geohydrology and Ground-Water Quality Beneath the 300 Area, Hanford Site, Washington. PNL-2949, Pacific Northwest Laboratory, Richland, Washington.

McMahon, W. J., and R. E. Peterson. 1992. Estimating Aquifer Hydraulic Properties Using the Ferris Method, Hanford Site, Washington. DOE/RL-92-64, Rev. 0, Westinghouse Hanford Company, Richland, Washington.

Runchal, A. K., and B. Sagar. 1989. PORFLO-3: A Mathematical Model for Fluid Flow, Heat, and Mass Transport in Variably Saturated Geologic Media. WHC-EP-0041, Westinghouse Hanford Company, Richland, Washington.

Schalla, R., R. W. Wallace, R. L. Aaberg, S. P. Airhart, D. J. Bates, J. V. M. Carlile, C. S. Cline, D. I. Dennison, M. D. Freshley, P. R. Heller, E. J. Jensen, K. B. Olsen, R. G. Parkhurst, J. T. Rieger, and E. J. Westergard. 1988. Interim Characterization Report for the 300 Area Process Trenches. PNL-6716, Pacific Northwest Laboratory, Richland, Washington. 


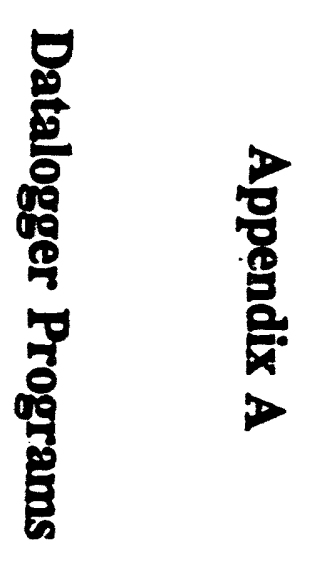
윰
뭇 


\section{Appendix A}

\section{Datalogger Programs}

Several datalogger programs were used to gather the required data. Each program combination is shown separately for reference. All programs begin with instructions to measure battery voltage and store it for display. Also, an instruction is included to measure with high resolution.

The widest use was made of the program to measure pressure. This program appears first. The second program adds thermocouple temperature to pressure. The third program replaces thermocouple temperature with a combination of solution electrical conductivity (EC) and temperature. The fourth program is for EC and temperature. 
Pressure Measurement Only

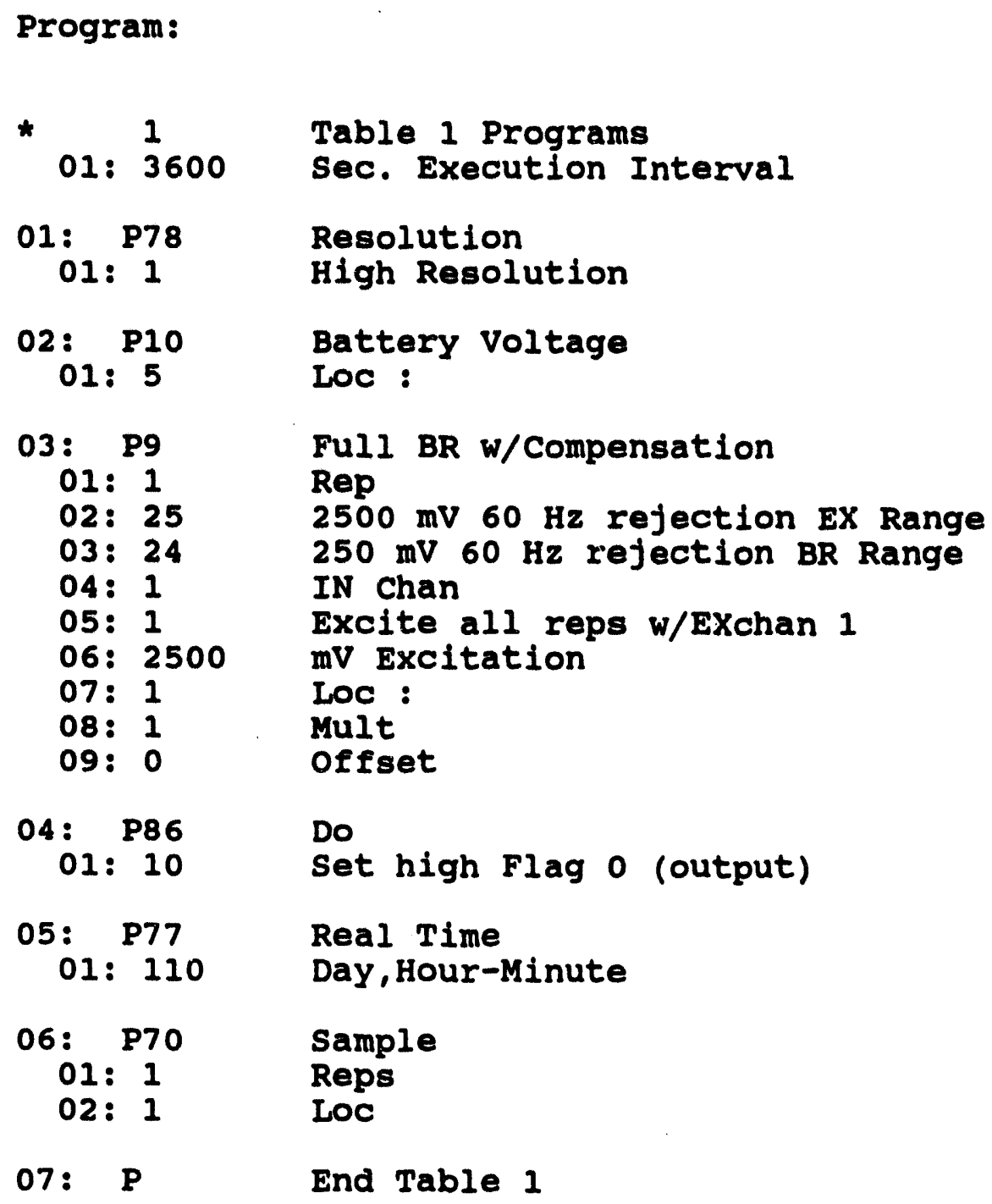

End Table 1 
Pressure and Thermocouple Temperature Measurement

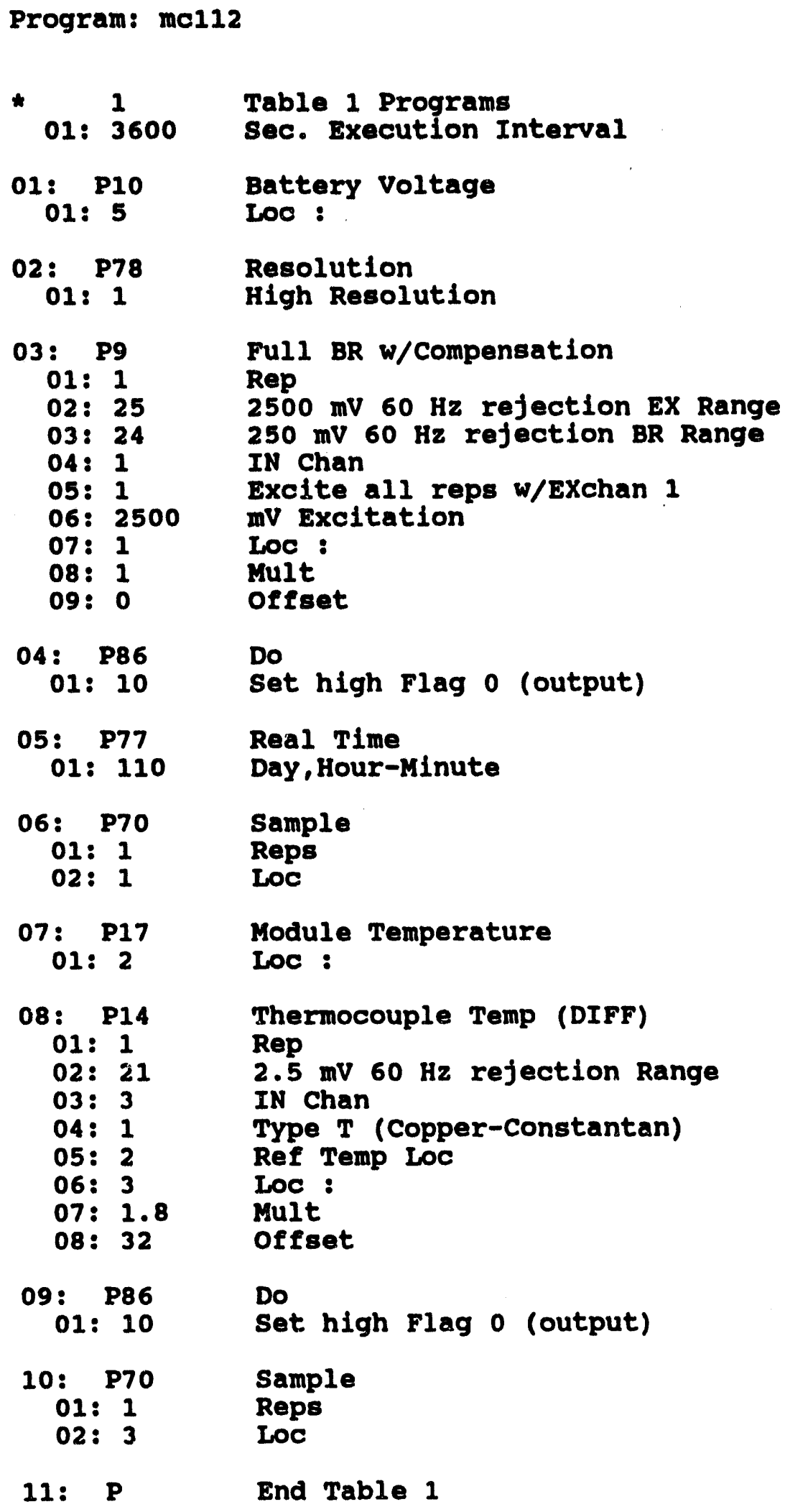

Do

Set high Flag 0 (output)

Real Time

Day, Hour-Minute

Sample

Reps

LOC

Module Temperature

Loc :

Thermocouple Temp (DIFF)

Rep

$2.5 \mathrm{mV} 60 \mathrm{~Hz}$ rejection Range

IN Chan

Type T (Copper-Constantan)

Ref Temp Loc

IOC :

Mult

offset

Do

Set high Flag 0 (output)

Sample

Reps

LOC

End Table 1

\section{A.3}


Pressure, Electrical Conductivity, and Temperature Measurement

Program: mc125

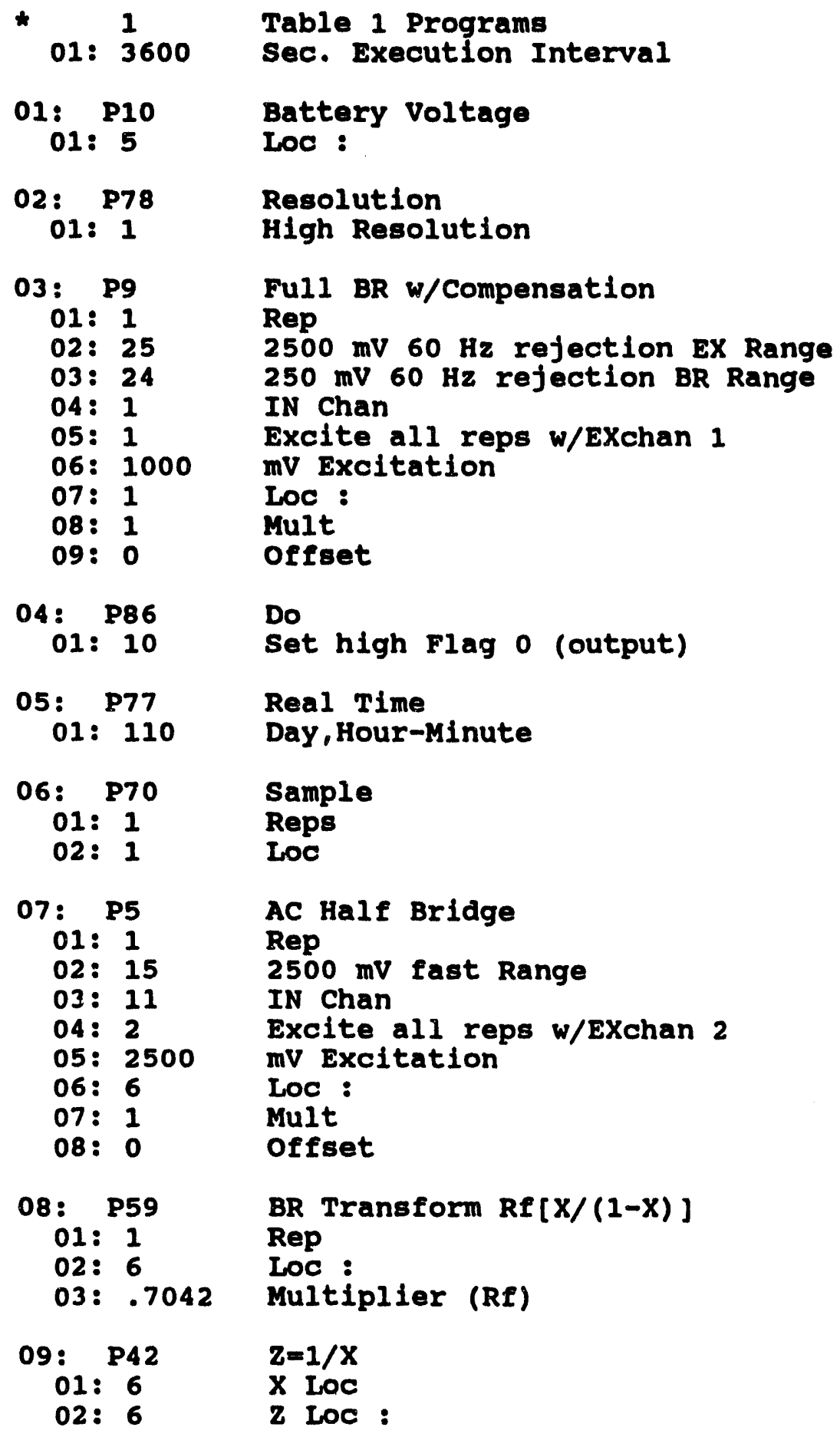

Do

Set high Flag 0 (output)

Real Time

Day, Hour-Minute

\section{A. 4}


Pressure, Electrical Conductivity, and Temperature Measurement (contd)

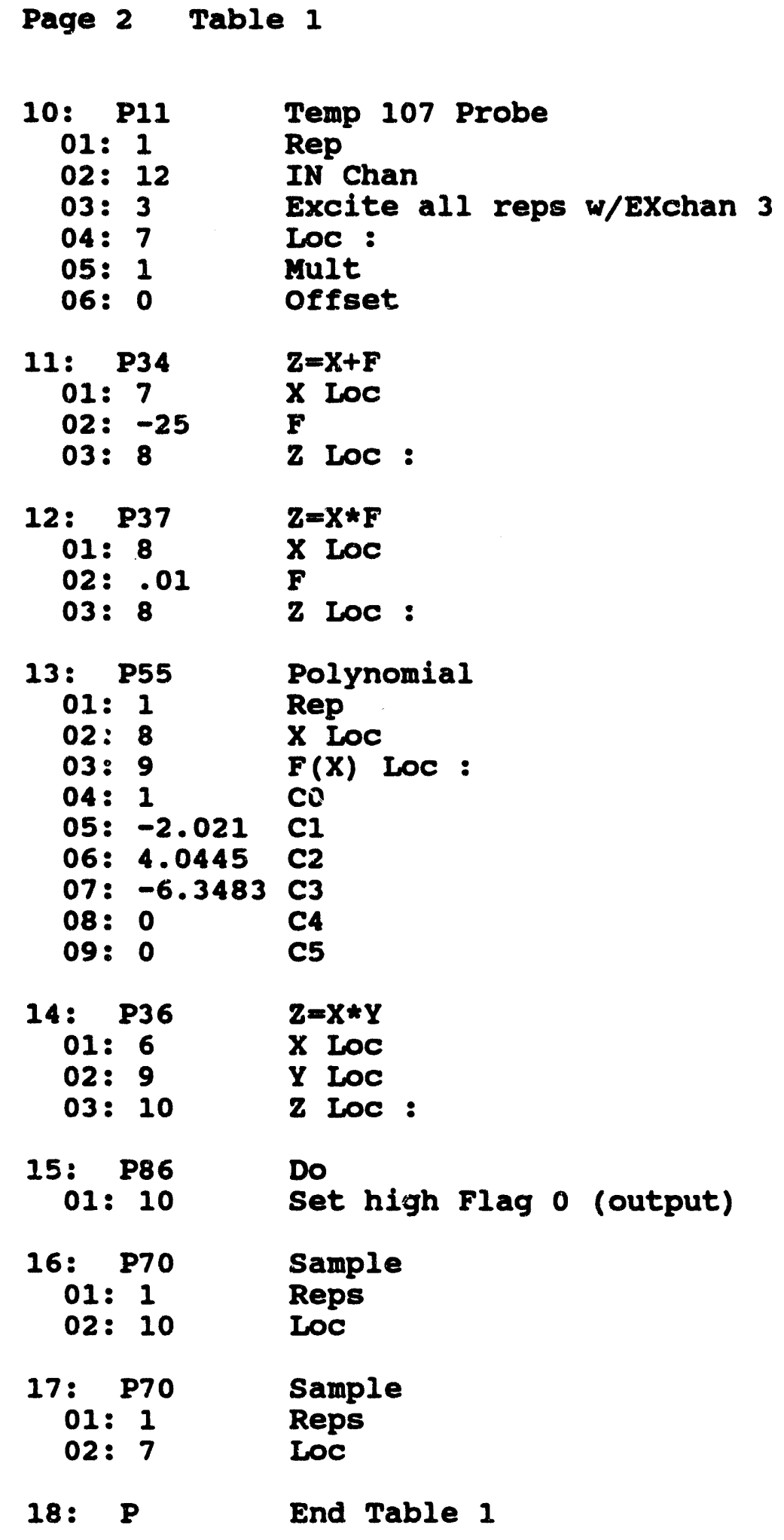

14: P36

01: 6

02: 9

03: 10

$z=X * Y$

$X$ LOC

$Y$ LOC

2 LOC :

$\begin{aligned} \text { 15: P86 } & \text { Do } \\ \text { 01: } 10 & \text { Set high Flag O (output) } \\ \text { 16: P70 } & \text { Sample } \\ \text { 01: } 1 & \text { Reps } \\ \text { 02: } 10 & \text { LoC } \\ \text { 17: P70 } & \text { Sample } \\ 01: 1 & \text { Reps } \\ 02: 7 & \text { LoC } \\ \text { 18: P } & \text { End Table 1 }\end{aligned}$


Electrical Conductivity and Temperature Measurements Only

Program: mc139

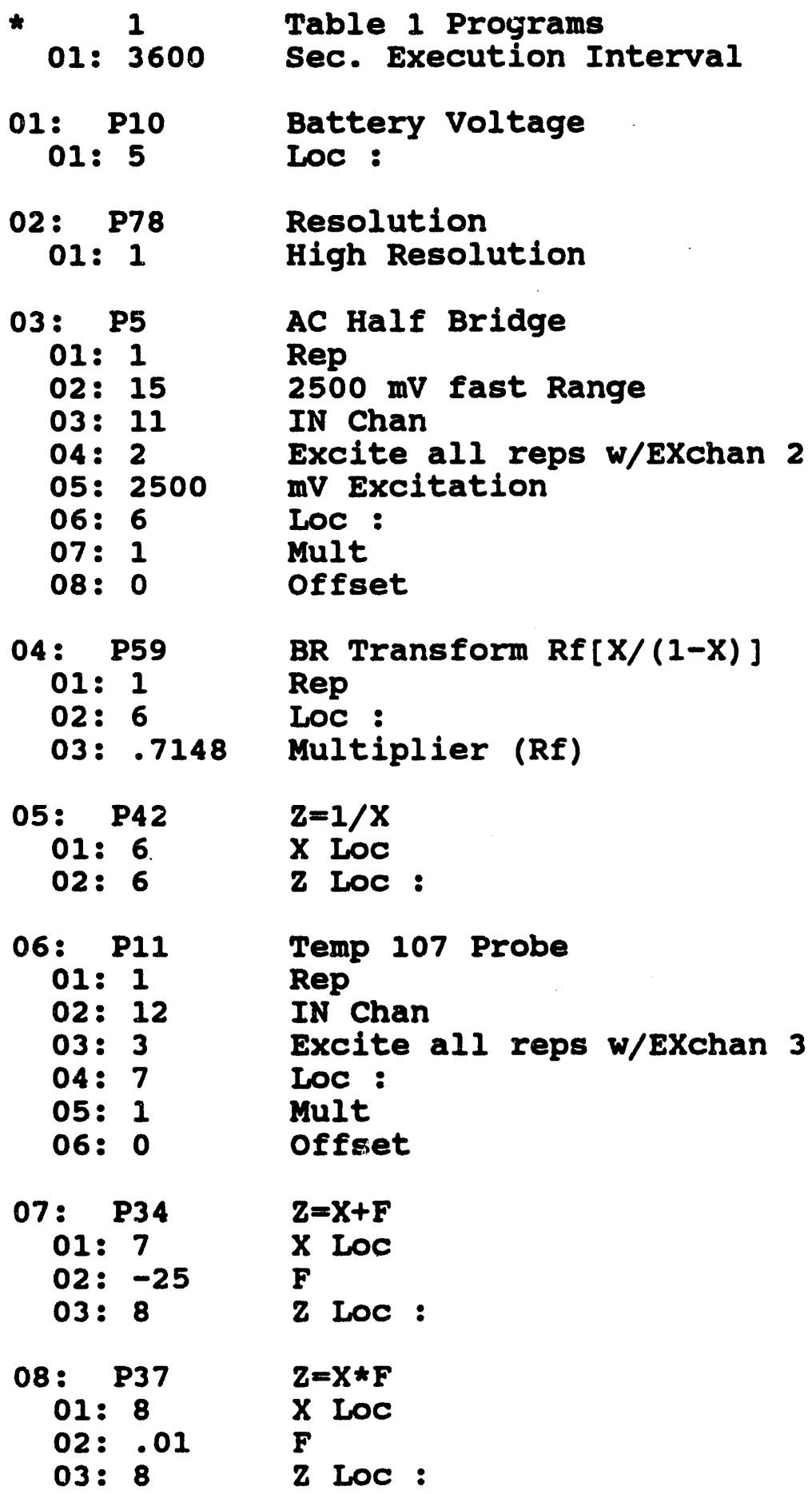


Electrical Conductivity and Temperature Measurements Only (contd)

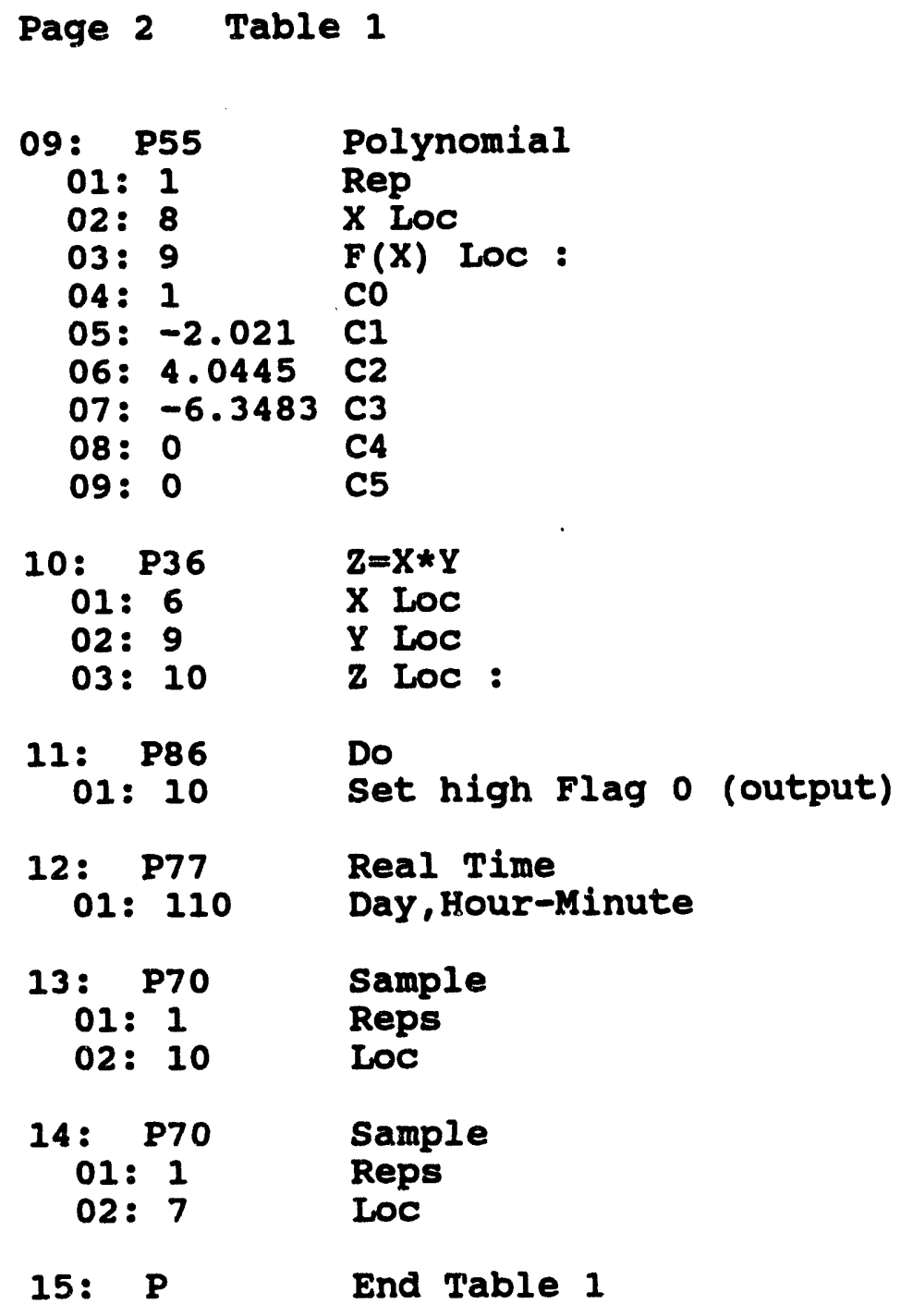

15: P

Do

Set high Flag 0 (output)

Real Time

Day, Hour-Minute

Sample

Reps

LOC

Sample

Reps

IOC

End Table 1 


\section{Appendix B}

\section{Steel Tape and Datalogger Paired Sets}




\section{Appendix B}

\section{Steel Tape and Datalogger Paired Sets}

Appendix B shows steel tape and datalogger paired sets that were used as data quality checks on the monitor system. Data from the initial sets in 1991 through December 1993 are included.

B.1 
Tape and Datalogger Reading Pairs

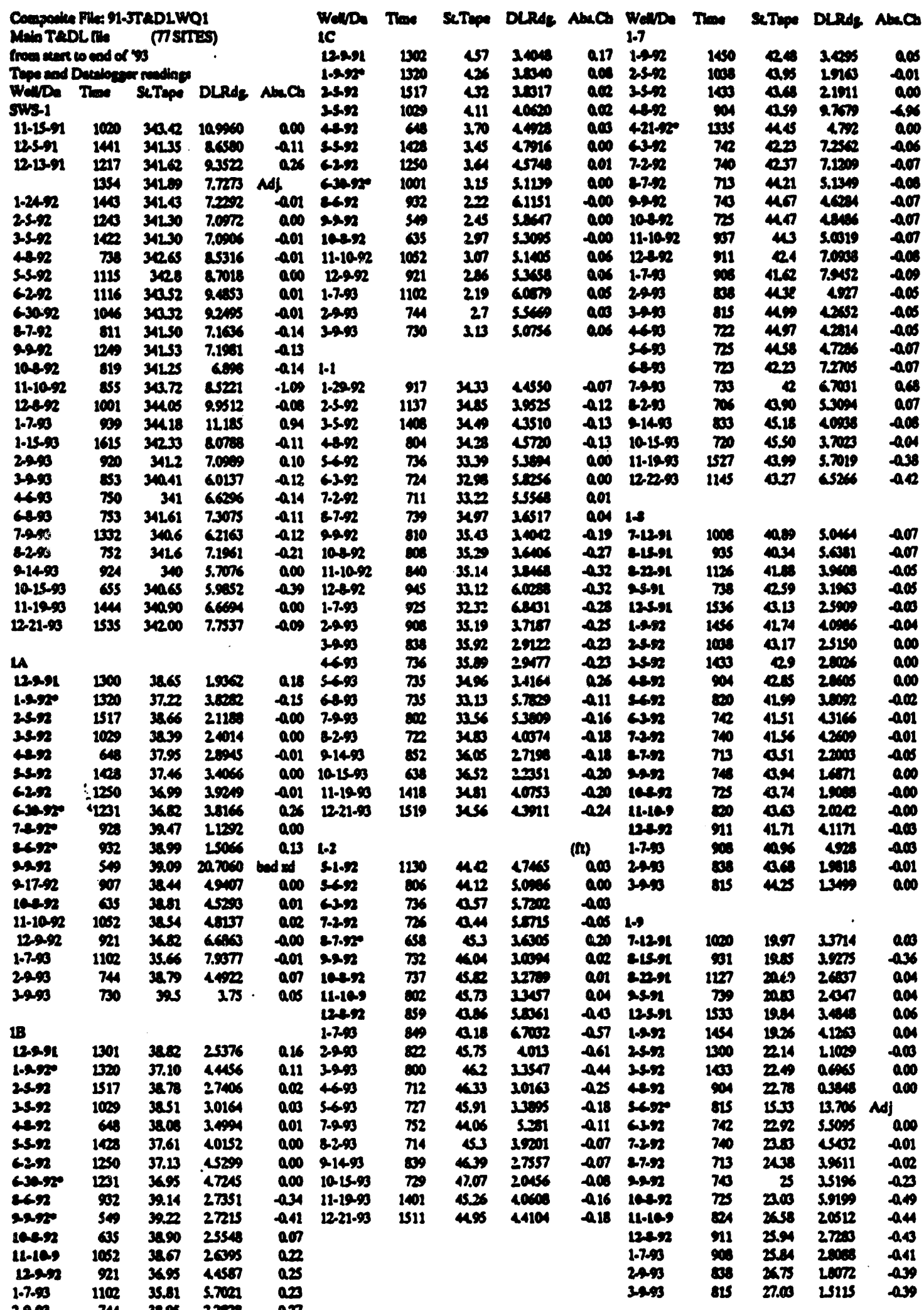


Tape and Datalogger Reading Pairs (contd)

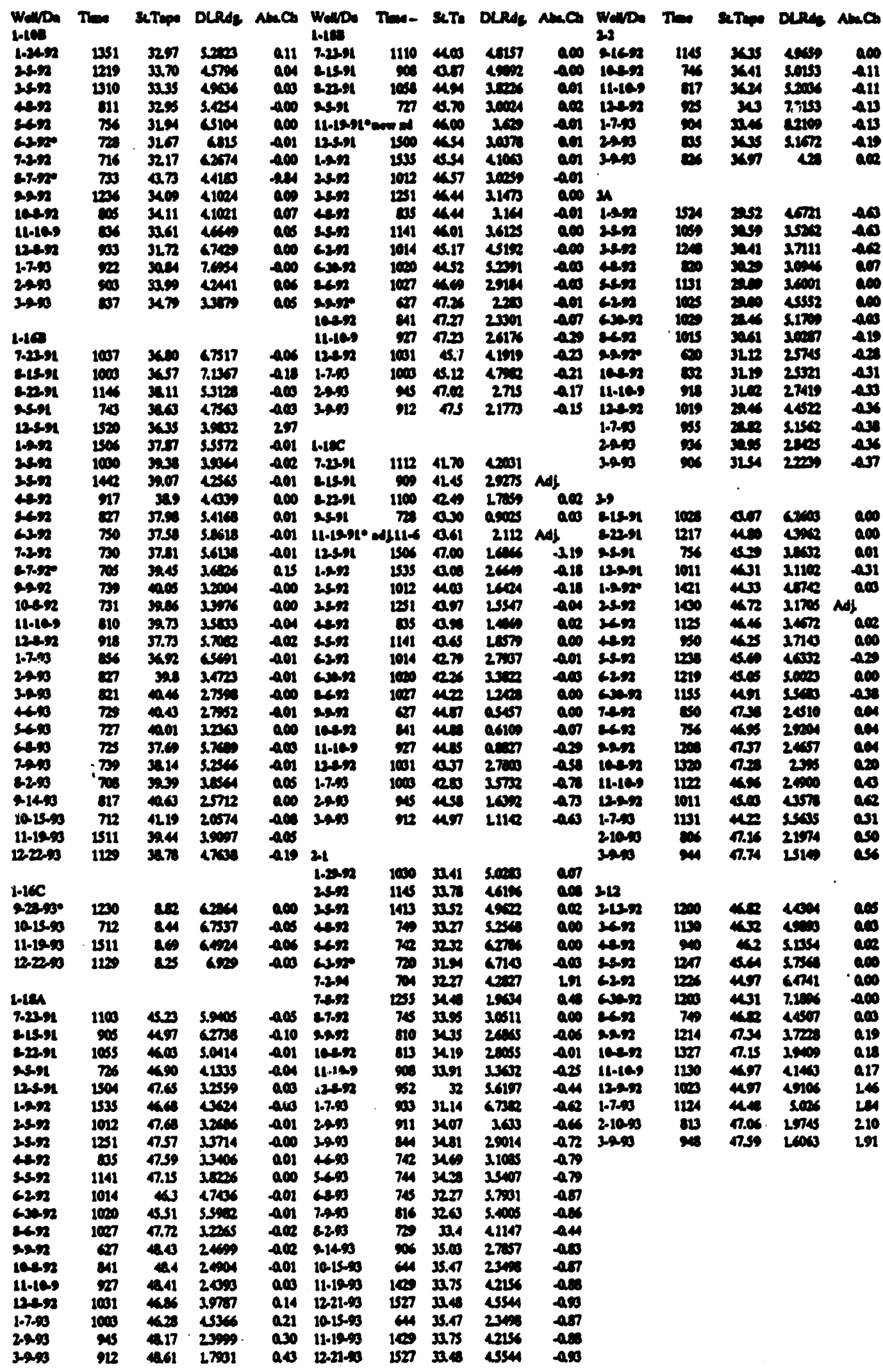

B. 3 
Tape and Datalogger Reading Pairs (contd)

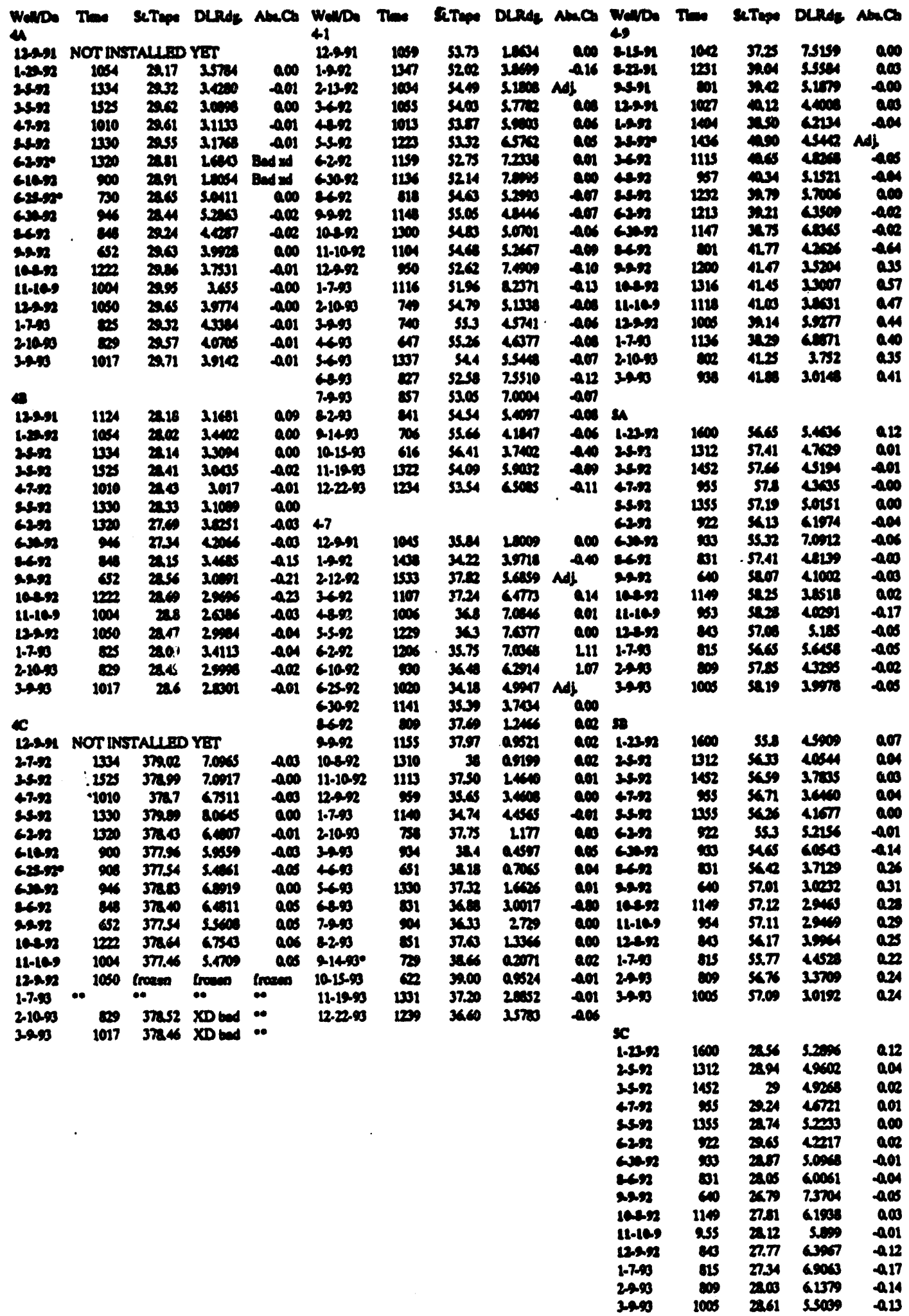


Tape and Datalogger Reading Pairs (contd)

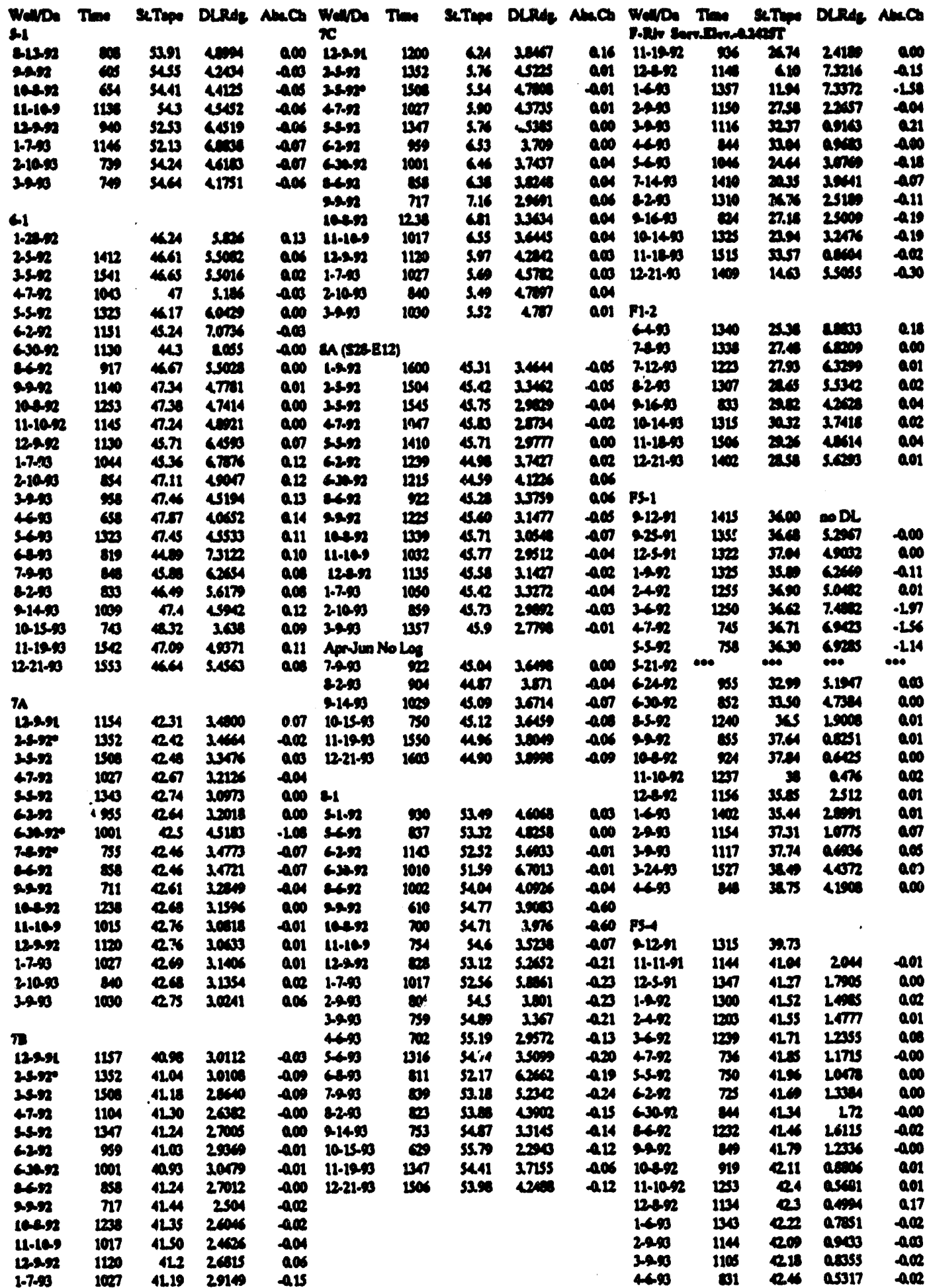


Tape and Datalogger Reading Pairs (contd)

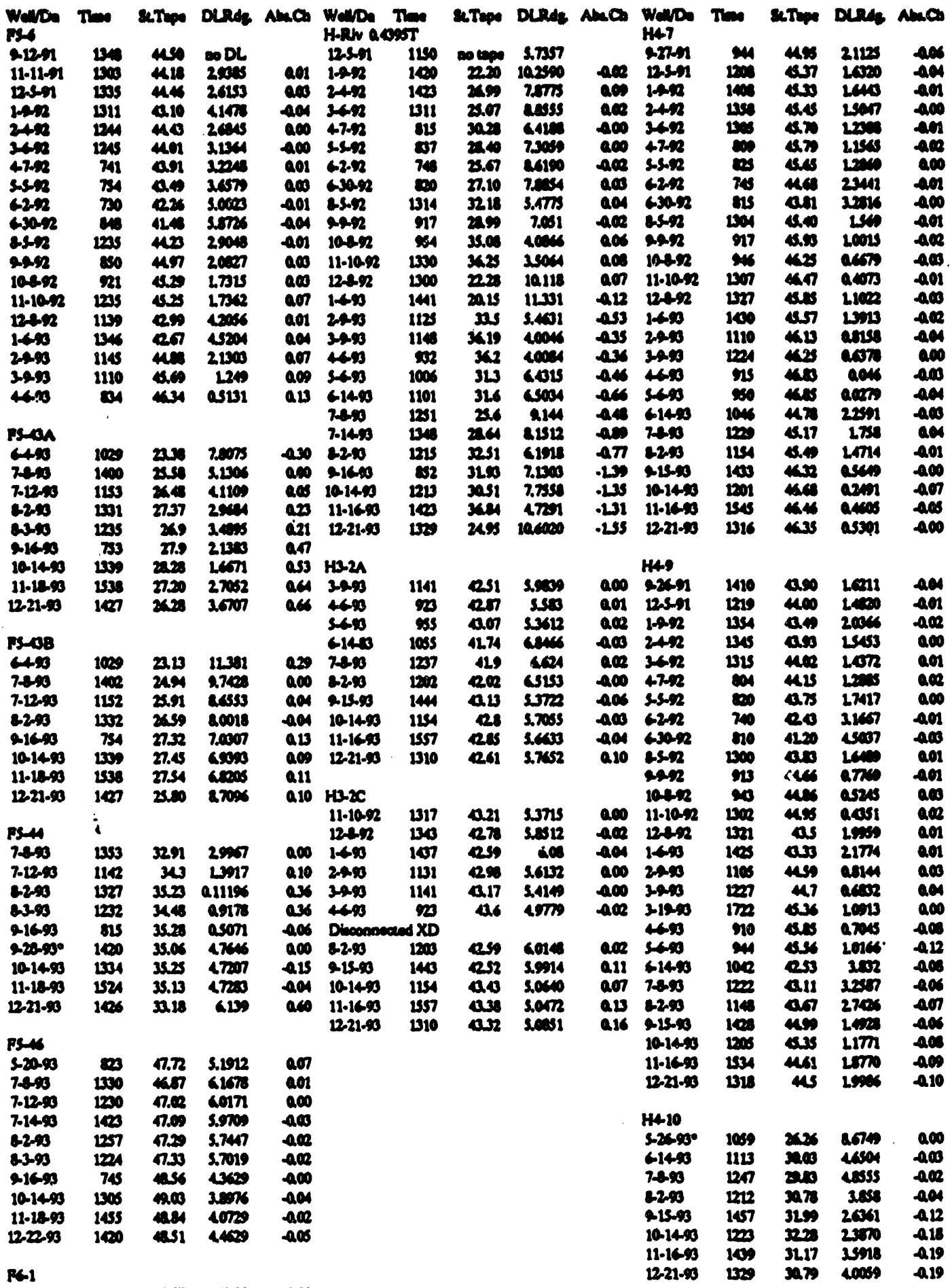


Tape and Datalogger Reading Pairs (contd)

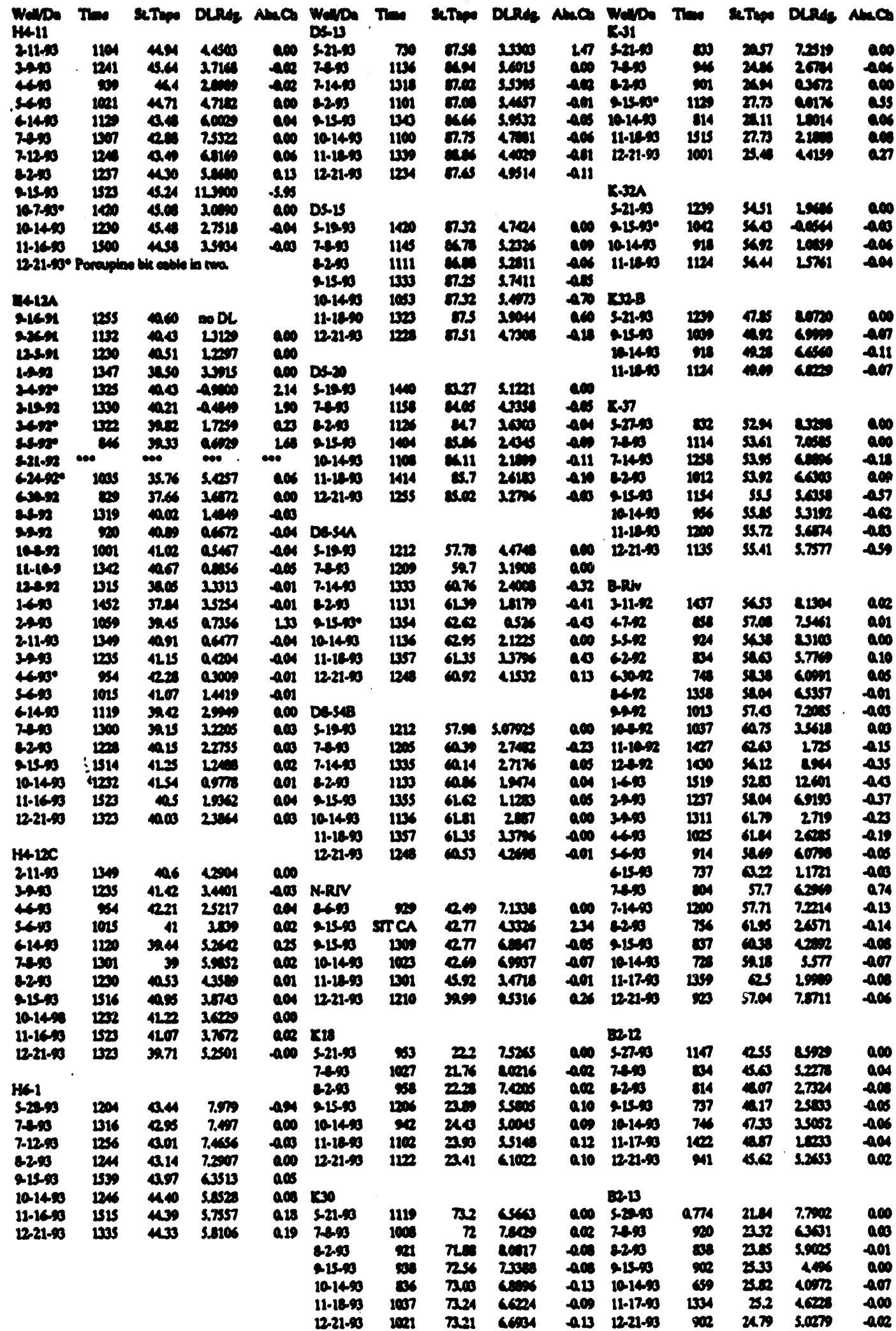

B.7 
Tape and Datalogger Reading Pairs (contd)

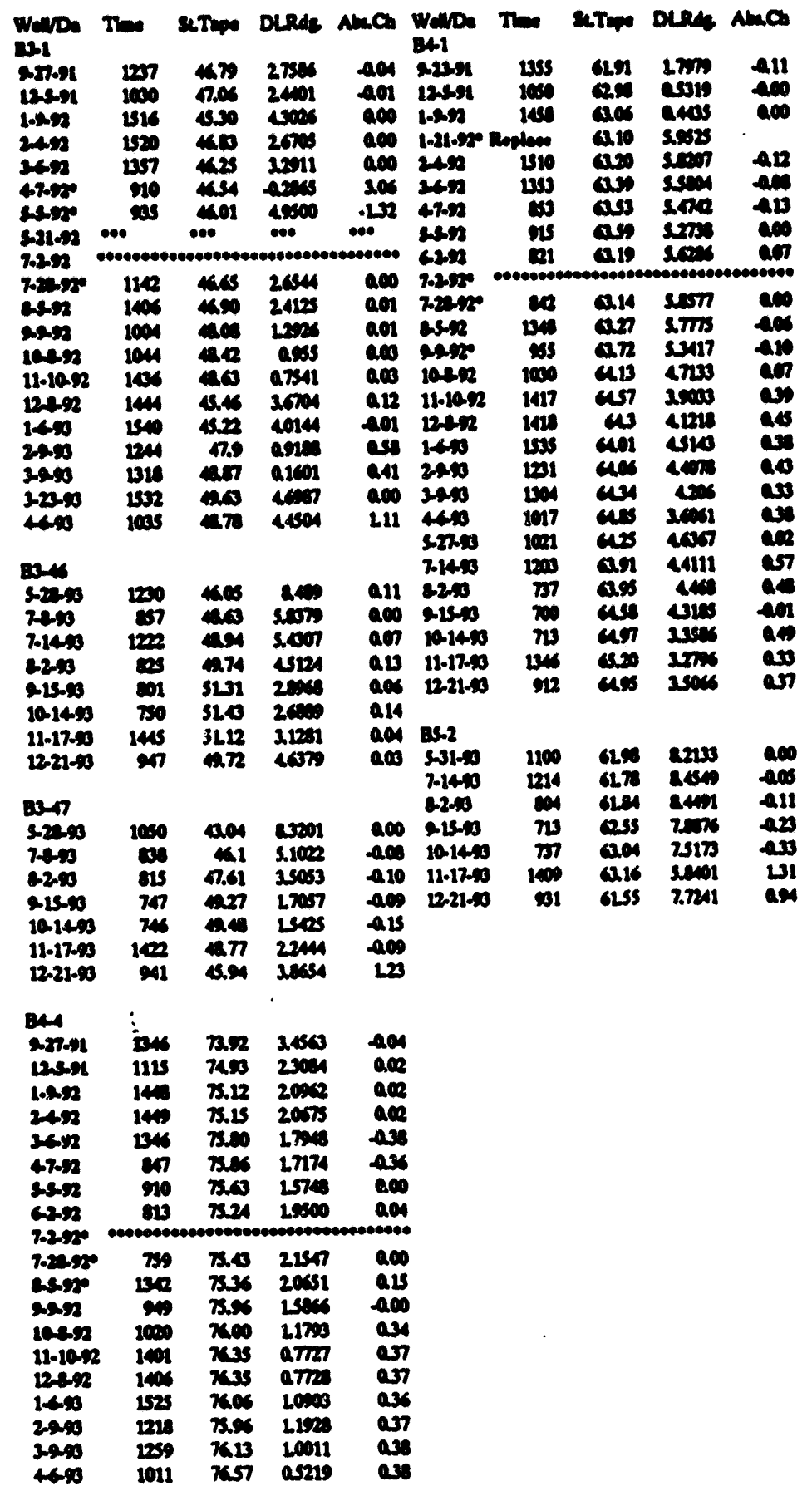




\section{Appendix C}

\section{Location and Calibration Data}




\section{Appendix C}

\section{Location and Calibration Data}

Appendix $\mathbf{C}$ contains information about the locations of the wells and river stations, their assigned station number, and the transducers used there. Next is the initial calibration data pertaining to each transducer.

C.1 


\section{Installation Data}

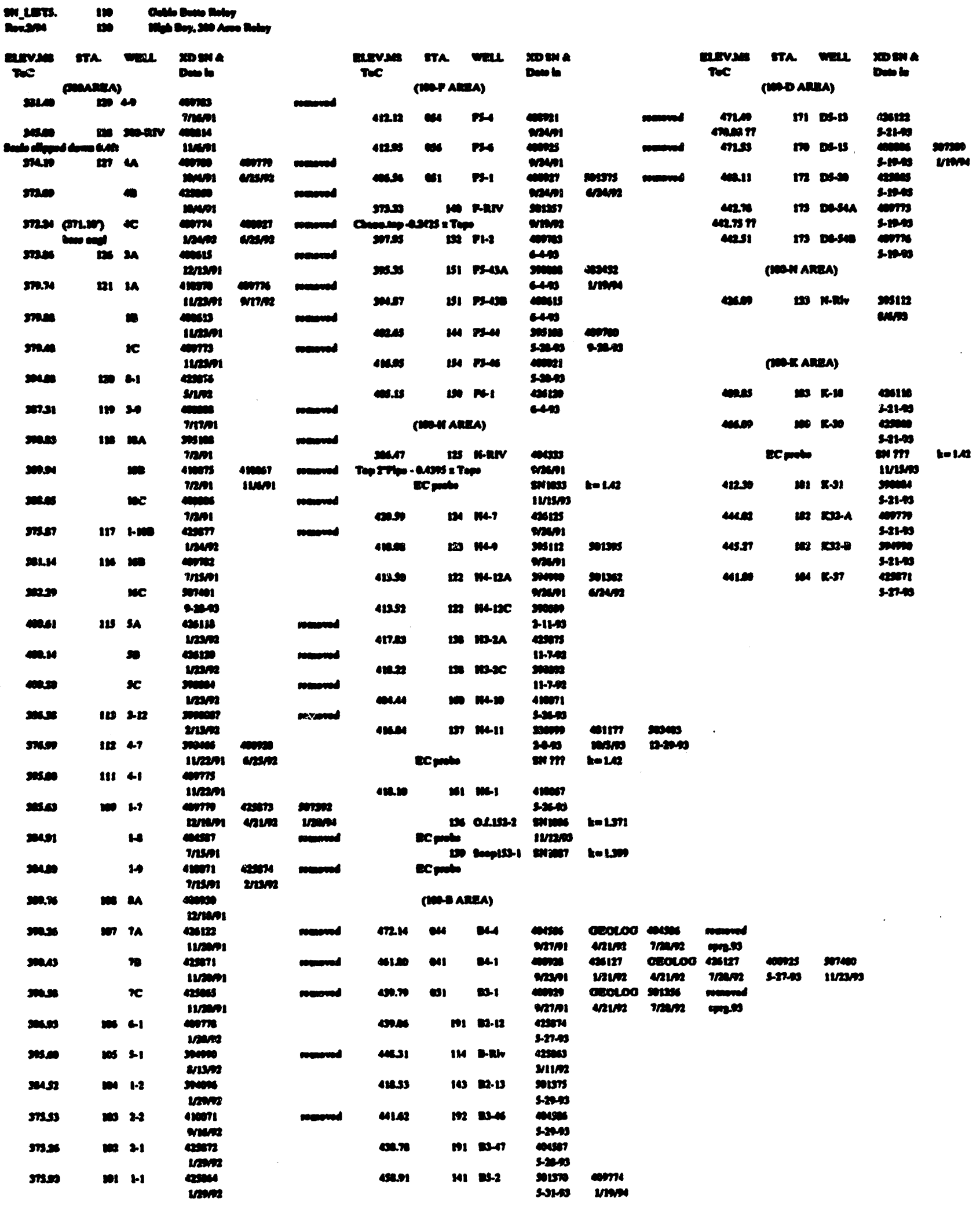

C. 2 
Initial Calibration Data

CALIBRATION FROM LRBSA110

\begin{tabular}{|c|c|c|c|c|c|c|c|}
\hline XDSN & Factor & Original & $\begin{array}{l}\text { Page } \\
\text { Linted } \\
\text { IN } 54110\end{array}$ & 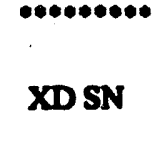 & Ori & & $\begin{array}{l}\text { Page } \\
\text { Lated } \\
\text { N } 54110\end{array}$ \\
\hline 306999 & 0.930129 & 49 & 57 & 425871 & 0.930162 & 34 & 55 \\
\hline 390008 & 0.929759 & 45 & 57 & 425872 & 0.925998 & 46 & 57 \\
\hline 390084 & 0.925817 & 44 & 56 & 425873 & 0.924778 & 53 & 58 \\
\hline 390088 & 0.921753 & & & 425874 & 0.927466 & 52 & 58 \\
\hline 390089 & 0.930984 & 50 & 57 & 425875 & 0.923511 & 55 & 58 \\
\hline 390092 & 0.933043 & 48 & 57 & 425876 & 0.933277 & 38 & 55 \\
\hline 390466 & 0.931572 & 32 & 43 & 425877 & 0.924719 recal & 54 & 58 \\
\hline 394096 & 0.940487 & 10 & 38 & 426118 & 0.919618 & 40 & 56 \\
\hline 394990 & 0.934299 & 2 & 38 & 426120 & 0.927198 & 41 & 56 \\
\hline 395108 & 0.928344 & 23 & 42 & 426122 & 0.927131 & 33 & 55 \\
\hline 395112 & 0.934208 & 5 & 38 & 426123 & 0.928045 & 43 & 56 \\
\hline 400613 & $0.928318 r$ & 25 & 42 & 426125 & 0.924634 & 39 & 56 \\
\hline 400615 & 0.926237 & 30 & 43 & 426127 & 0.92888 recal & 42 & 56 \\
\hline 400806 & 0.925422 & 22 & 41 & REPAIREO & X's's added $94-92$ & & \\
\hline 400808 & 0.924320 & 26 & 42 & 304980 & 0.024224 & 3 & 108 \\
\hline 400814 & 0.934277 & 24 & 42 & 400927 & 0.010460 & 1 & 108 \\
\hline 400921 & 0.928259 & 6 & 38 & 400928 & 0.824320 & 4 & 108 \\
\hline 400925 & 0.938506 & 4 & 38 & 400779 & 0.930846 & 5 & 108 \\
\hline 400927 & 0.925948 & 3 & 38 & 410071 & 0.029051 & 2 & 108 \\
\hline 400928 & 0.930894 & 9 & 38 & NEW XD's & added 0-4-92 & & \\
\hline 400929 & 0.937396 & 7 & 38 & 501356 & 1.056266 & 3 & 108 \\
\hline 400930 & 0.928236 & 11 & 40 & 501357 & 1.050504 & 4 & 108 \\
\hline 404333 & 0.934862 & 1 & 38 & 501350 & 1.008546 & 2 & 108 \\
\hline 404586 & 0.932610 & 8 & 38 & 501362 & 1.058788 & 5 & 108 \\
\hline 404587 & 0.927600 & 12 & 40 & 501370 & 1.049868 & 1 & 108 \\
\hline 409773 & 0.933826 & 17 & 41 & 501375 & 1.050638 & 6 & 108 \\
\hline 409774 & 0.9307 rec & 15 & 40 & NEW XD's & added - & & \\
\hline 409775 & 0.930978 & 28 & 42 & 481177 & 1.053180 & & 147 \\
\hline 409776 & 0.933341 & 27 & 42 & 482452 & $1.05108 \mathrm{recal}$ & & 146 \\
\hline 409778 & 0.931615 & 16 & 40 & 495533 & 1.051341 & & 146 \\
\hline 409779 & 0.935513 & 13 & 40 & 500042 & 1.051024 recal & & 146 \\
\hline 409780 & 0.927762 & 29 & 43 & 501370 & $1.05512 \mathrm{recal}$ & & 146 \\
\hline 409782 & 0.930449 & 31 & 43 & 503408 & 1.052781 & & 147 \\
\hline 409783 & 0.928530 & 14 & 40 & 503548 & 1.058283 & & 146 \\
\hline 410067 & 0.931645 & 20 & 41 & 507381 & 1.087470 recal & & 146 \\
\hline 410070 & 0.931287 & 18 & 41 & 507384 & $1.047854 \mathrm{recal}$ & & 147 \\
\hline 410071 & 0.931156 & 19 & 41 & 507389 & $1.038989 \mathrm{recal}$ & & 147 \\
\hline 410075 & 0.923157 & 21 & 41 & 507302 & $1.068226 \mathrm{recal}$ & & 147 \\
\hline 425860 & 0.925743 & 36 & 55 & 507394 & 1.055505 recal & & 146 \\
\hline 425863 & 0.926984 & 51 & 58 & 507396 & 1.082276 reoal & & 147 \\
\hline 425864 & 0.929513 & 47 & 57 & 607397 & 1.055673 recal & & 146 \\
\hline 425865 & 0.932000 & 35 & 55 & 507400 & 1.051118 & & 147 \\
\hline 425871 & 0.930162 & 34 & 55 & 507401 & 1.053842 & & 146 \\
\hline
\end{tabular}




\section{Distribution}

No. of

Copies

\section{OEFSITE}

2 DOE/Office of Scientific and Technical Information

\section{ONSITE}

9 DOE Richland Operations Office

B. L. Foley

M. J. Furman

G. I. Goldberg

E. D. Goller

R. D. Hildebrand

M. P. Johansen (USACE)

R. G. McLeod (2)

K. M. Thompson

33 Westinghouse Hanford Company

R. A. Carison

J. A. Coates

D. A. Duranceau

K. R. Fecht

L. C. Hulstrom (10)

W. J. McMahon

R. E. Peterson (10)

J. W. Roberts

K. R. Simpson

J. C. Sonnichsen

R. L. Biggerstaff

ERC (2)

EDMC (2)
A5-19

R3-80

A5-19

A5-19

A5-55

A5-19

A5-19

A5-15

H6-03

H6-06

H4-14

H6-06

H6-03

H6-06

H6-06

H6-02

H6-06

H4-14

H6-02

H6-07

H6-08
No. of

Copies

22 Pacific Northwest Laboratory

M. P. Bergeron

K6-77

J. V. Borghese

K6-96

M. D. Campbell (10)

D. R. Newcomer

K6-77

R. Schalla

F. A. Spane

Publishing Coordination

Technical Report Files (5)

Routing

R. M. Ecker

SE-VI

M. J. Graham

K6-78

P. M. Irving

K6-98

C. S. Sloane

K6-04

P. C. Hays (last)
K6-96

K6-96

Dist.1 


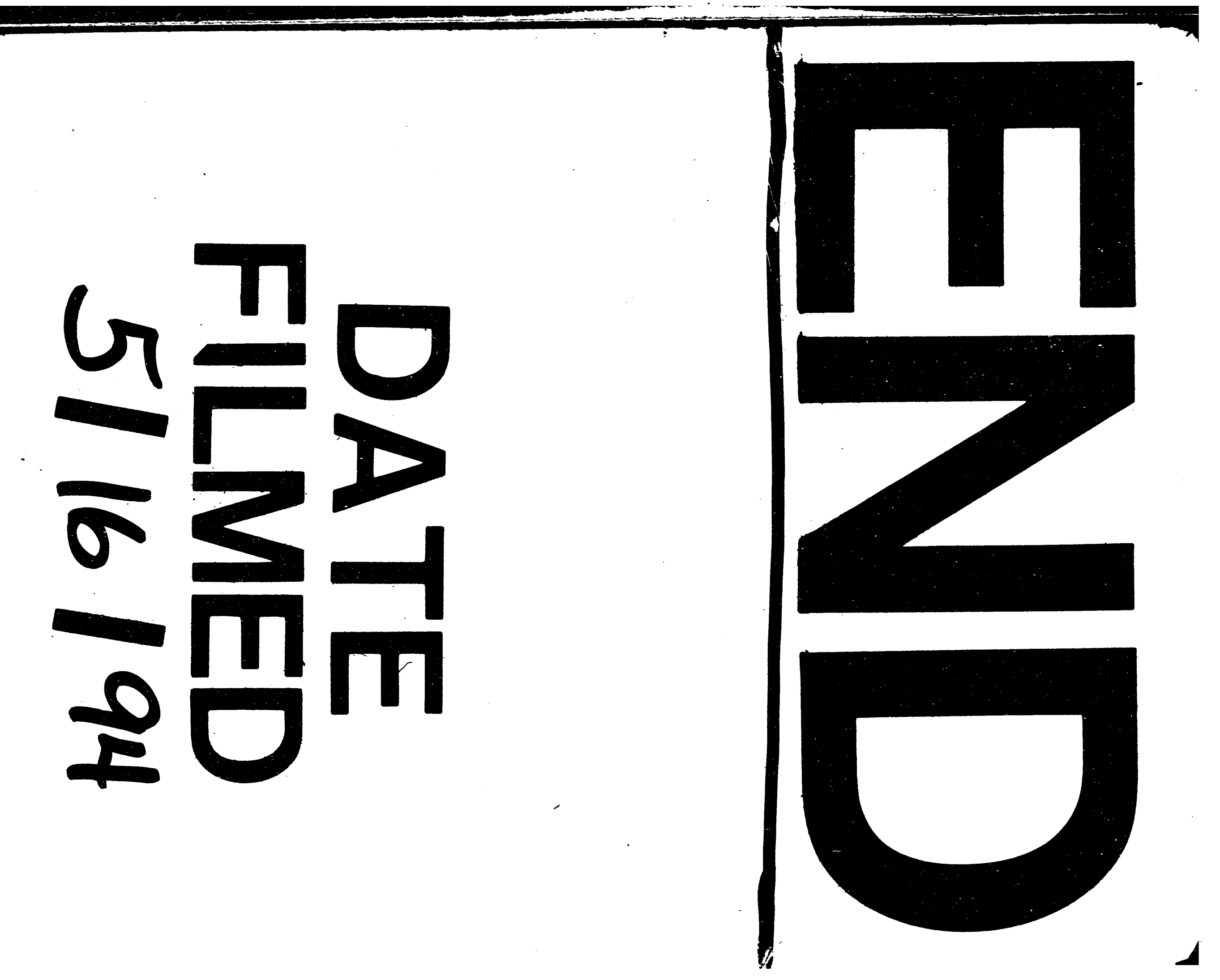




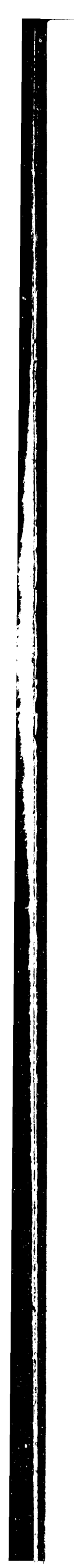

